\title{
फु
}

\section{Global simulations of Minkowski spacetime including spacelike infinity}

\author{
Georgios Doulis ${ }^{1, *}$ and Jörg Frauendiener ${ }^{2,3, \dagger}$ \\ ${ }^{1}$ Max Planck Institute for Gravitational Physics (Albert-Einstein-Institute) \\ ${ }^{2}$ Department of Mathematics and Statistics, University of Otago, Dunedin 9010, New Zealand \\ ${ }^{3}$ Department of Mathematics, University of Oslo, Oslo 0316, Norway \\ (Received 14 September 2016; published 30 January 2017)
}

In this work, we study linearized gravitational fields on the entire Minkowski spacetime including spacelike infinity. The generalized conformal field equations linearized about a Minkowski background are utilized for this purpose. In principle, this conformal representation of Einstein's equations can be used to carry out global simulations of Minkowski spacetime. We thoroughly investigate this possibility.

DOI: 10.1103/PhysRevD.95.024035

\section{INTRODUCTION}

The recent direct observation of gravitational waves [1] demonstrated conclusively the predictive power of numerical relativity in problems of high complexity where the known analytical methods cannot be applied. Since there are no analytical expressions for the waveforms expected from binary black hole mergers, like the one observed by LIGO, they were obtained numerically and then compared to the actual findings of [1]. The fact that the observed waveforms agree to a high level of accuracy with the theoretically expected ones marks another success of general relativity.

Numerical relativity experienced a rapid advance in the last couple of decades, fueled mainly by the urge to model binary systems of massive compact objects. The standard way of describing a system of this kind is as an isolated system, i.e., a compact self-gravitating astrophysical object embedded in an asymptotically flat spacetime. Although this approximation provides a rigid basis for the analytical and numerical study of self-gravitating systems, it puts a huge burden especially on the numerical side since now the infinite span of the asymptotically flat spacetime must be somehow simulated with finite computational resources. Several approaches have been developed over the years to deal with this problem.

The most common approach is based on the Cauchy formulation of Einstein's equations. In this approach the spacelike hypersurfaces of constant time are truncated at a finite distance where an artificial timelike boundary is introduced. To ensure that the resulting initial boundary value problem is mathematically well posed and numerically stable, appropriate boundary conditions must be imposed at this boundary. The boundary conditions must satisfy the constraints on every time slice and must be absorbing, i.e., purely outgoing, in order to minimize the

\footnotetext{
gdoulis@phys.uoa.gr

joergf@maths.otago.ac.nz
}

amount of spurious reflections on the boundary. During the last decade, following the seminal works [2-4], more and more sophisticated codes were developed based on this approach. An indication of the high quality of these numerical schemes is the fact that the numerically computed waveforms with which the actual waveforms detected by LIGO [1] have been compared were modeled upon [3]. Despite its successes, and the fact that there is definitely space for further improvement, the Cauchy approach has certain limitations emanating mainly from the fact that, in general relativity, local expressions for the gravitational energy density and flux do not exist. This in turn makes it impossible to construct completely absorbing boundary conditions for boundaries that stand at a finite distance. In addition, as shown in [5], when gravity is coupled to (e.g., scalar or Yang-Mills) fields, the location where information is extracted from is of essential physical importance as the decay rates of the fields depend on the location of the observer. One also has to bear in mind that while the Cauchy approach is very well adapted to the study of binary and other isolated systems, it cannot be used to answer questions that require the inclusion of spacetime infinity into the computational domain like the feasibility of global simulations of entire spacetimes, the stability of black hole spacetimes, and the cosmic censorship conjecture. Strictly speaking, even the aim of computing the exact gravitational waveforms emitted from an isolated system necessitates the inclusion of the entire spacetime. However, for the accuracies needed for the current detectors, this does not seem to be an issue.

A possible way to extend the standard Cauchy approach all the way out to infinity is by combining it with the so-called characteristic approach [6,7]. This approach uses lightlike, instead of spacelike, hypersurfaces that reach all the way out to null infinity through a compactification of the spatial coordinate. In the resulting Cauchy-characteristic matching method [8], the interior region is treated with the standard Cauchy techniques, while the distant asymptotically flat 
region is left to the characteristic approach. (In [9] the characteristic method has also been used successfully to treat the inner boundary of the Cauchy domain in a similar way with the outer boundary.) The two approaches are matched along a transparent, continuous (and at least once differentiable) timelike boundary that has the dual role of providing an outer (inner) boundary condition for the Cauchy (characteristic) evolution. The data flow at the boundary is a two-way flow with the Cauchy and characteristic codes providing exact boundary values for each other. The construction of such a two-way boundary is not so trivial as the whole (analytical and numerical) setup changes at the interface. This complexity calls for the development of highly sophisticated techniques that could enable the construction of such a boundary. Although considerable progress has been made in that direction [10], the current implementation of the boundary is still one way [11]. Specifically, absorbing boundary conditions are imposed on the interface between the two codes with data flowing only from the Cauchy to the characteristic region, but not vice versa. Thus, in its current status the Cauchy-characteristic matching suffers from the same limitations as the standard Cauchy method.

It seems that the inclusion of infinity into the computational domain demands a more sophisticated approach than just the introduction of a boundary at finite distance. Penrose's concept of conformal infinity [12] provides an unexpectedly simple and mathematically sound way to deal with the difficulties related to the infinite span of asymptotically flat spacetimes. In this picture the spacetime metric $\bar{g}$ is conformally transformed, $\bar{g}=\Omega^{-2} g$, with an appropriately chosen conformal factor $\Omega$. In this way spacetime infinity is brought to a finite distance. In the conformal spacetime $g$, the outer boundary of the computational domain (that now lies at infinity) emerges naturally as the locus where $\Omega=0$, and thus it does not have to be artificially introduced. In addition, as it lies at infinity, it is completely absorbing, and thus no boundary conditions have to be imposed there. Research in this setting has taken two different but closely related directions.

In the first approach the spacetime is foliated with spacelike hyperboloidal hypersurfaces that reach null infinity in order to avoid the singular nature of spacelike infinity. A number of different formulations of Einstein's equations in the context of the hyperboloidal foliation have appeared through the years. In [13] Friedrich managed to express Einstein's equations for the conformal metric as a manifestly symmetric hyperbolic system that is regular all the way to null infinity. With this set of equations, hyperboloidal initial data have been successfully evolved along null infinity, and even up to timelike infinity, in several different scenarios [14-16]. An alternative approach by Moncrief and Rinne [17] employs the standard ADM formulation to express the conformally transformed Einstein equations on hyperboloidal hypersurfaces of constant mean curvature. Although formally singular, the resulting equations are actually regular at null infinity provided that certain regularity conditions hold there. Based on this formulation, long-term stable numerical evolution was achieved in axial and spherical symmetry with $[18,19]$ and without [20] matter. Recently, the first extensive numerical implementation [21] of yet another formulation of Einstein's equations on constant mean curvature slices by Zenginoğlu [22] has appeared with very encouraging results.

The main motivation for using hyperboloidal hypersurfaces is the avoidance of spacelike infinity. Thus, any approach based on a hyperboloidal foliation is only capable of evolving data along future null infinity and not along past null infinity as this would require going through the singular spacelike infinity. So, global simulations are not possible in this approach. Another implication of excluding spacelike infinity is that phenomena, such as scattering of gravitational waves, related to the inflow of gravitational radiation from null infinity cannot be studied. To address such questions, spacelike infinity must be brought into the picture. Recently, it was proposed in [23] that this could be done by extending the hyperboloidal approaches [17] in a way such that spacelike infinity is taken into account. The basic idea is to use a standard Cauchy evolution scheme to obtain data on a first hyperboloidal slice by evolving initial data that extend to spacelike infinity and then use these data as initial data for an already existing hyperboloidal code [20]. The feasibility of this idea crucially depends on the possibility of constructing Cauchy initial data that are static in the neighborhood of spacelike infinity and nonstatic in the interior. The static character of the data close to spacelike infinity guarantees that during the short Cauchy evolution, the boundary condition imposed on the outer boundary, which is placed well inside the static region, is exact. These kinds of initial data have recently been constructed in [23,24].

Another way of introducing spacelike infinity into the conformal picture is by employing the so-called generalized conformal field equations presented by Friedrich in [25]. In this approach the spacetime is foliated with generic spacelike hypersurfaces and Einstein's equations are reformulated as a symmetric hyperbolic system that is regular at spacelike and null infinity. The basic ingredient of this approach is the blowing up of spacelike infinity to a cylinder $I=[-1,1] \times \mathbb{S}^{2}$ of finite length that serves as a link between past and future null infinity. An extremely pleasant feature of the generalized conformal field equations is that they reduce to an intrinsic set of evolution equations on $I$; i.e., $I$ is a total characteristic of the system, and thus no boundary conditions are needed there. In addition, although high in number, the generalized conformal field equations have an extremely simple form as the majority of them are ordinary differential equations and the rest can be written in a symmetric hyperbolic form. The first attempt to implement this approach numerically 
appeared in [26] for asymptotically flat, axisymmetric, radiative spacetimes. The Otago relativity group followed with an extensive study of the behavior of linearized gravitational fields on a Minkowski background [27-31]. Therein, a stable and convergent code was developed that can successfully evolve several different types of initial data along (and in the neighborhood of) the cylinder $I$. Recently, the generalized conformal field equations have been used to compute a simulation of the response of a Schwarzschild black hole to the impact of gravitational waves [32] on a computational domain that includes the interior of the horizon and parts of null infinity, but not $I$.

In the present work the generalized conformal field equations [25] will be used to study gravitational perturbations on Minkowski spacetime, not only in the neighborhood of spacelike infinity but also on the entire Minkowski spacetime $\mathbb{M}$. To do so, we first conformally embed $\mathbb{M}$ into the Einstein static universe $\mathbb{E}$ (see Sec. II A). Then, in Sec. II B, by appropriately rescaling this finite representation of $\mathbb{M}$, spacelike infinity is blown up to a cylinder in accordance with Friedrich's construction. But now the range of the equations covers the entire Minkowski spacetime and not only the regions close to $I$. Since we explicitly make use of the spherical symmetry of the underlying Minkowski spacetime by using polar coordinates, some terms of the generalized conformal field equations are now singular at the origin (see Sec. III). We express them as a system of first-order (Sec. III A) and second-order (Sec. III B) partial differential equations and choose to implement numerically the latter as it behaves better numerically [33]. In order to provide initial data for the latter and to guarantee that the solutions they provide are the same, a correspondence is established between the two systems in Sec. III C. In Sec. IV B we describe the way in which the singular terms of the system (3.21) have been implemented at the origin. The above setting, in principle, could be used to carry out global simulations of $\mathbb{M}$ if the expected degeneracy of the evolution equations at the interface of $I$ with null infinity $\mathcal{I}$ could somehow be circumvented. The conformal compactification of Fig. 2(b) resulting from the rescaling (2.16) could serve this purpose. This possibility is thoroughly investigated in Sec. IV D 2.

\section{MINKOWSKI SPACETIME}

In this section we discuss the details of the setting in which Minkowski spacetime will be used in the following. The finite representation of Minkowski spacetime as part of the Einstein static universe will be briefly presented, and the blowing up of spacelike infinity to a finite cylinder pioneered in [25] will be described.

\section{A. Conformal compactification}

In the present work we perform a similar conformal transformation and coordinate change as we did in $[27,28]$.
However, this time we are not only interested in the neighborhood of spacelike infinity $i^{0}$ but also in the entire Minkowski space $\mathbb{M}$. We do not perform a coordinate inversion to get $i^{0}$ to be the new origin, but we use the wellknown conformal embedding of $\mathbb{M}$ into the Einstein static universe $\mathbb{E}$ to obtain a finite representation of $\mathbb{M}$.

Recall that $\mathbb{E}$ is the manifold $\mathbb{R} \times \mathbb{S}^{3}$ with metric

$$
g_{E}=\mathrm{d} T^{2}-\mathrm{d} R^{2}-\sin ^{2} R\left(\mathrm{~d} \theta^{2}+\sin ^{2} \theta \mathrm{d} \phi^{2}\right),
$$

where the coordinates range in the intervals

$$
\begin{aligned}
-\infty & <T<\infty, \quad 0<R<\pi, \quad 0<\theta<\pi, \\
-\pi<\phi<\pi . &
\end{aligned}
$$

Following [34], we briefly show how the entire Minkowski spacetime can be conformally compactified and represented as a finite part of the Einstein static universe. Our starting point will be the Minkowski metric expressed in spherical coordinates

$$
\bar{g}_{M}=\mathrm{d} t^{2}-\mathrm{d} r^{2}-r^{2}\left(\mathrm{~d} \theta^{2}+\sin ^{2} \theta \mathrm{d} \phi^{2}\right),
$$

which, when expressed in advanced $u=t+r$ and retarded $w=t-r$ null coordinates, becomes

$$
\bar{g}_{M}=\mathrm{d} u \mathrm{~d} w-\frac{1}{4}(u-w)^{2}\left(\mathrm{~d} \theta^{2}+\sin ^{2} \theta \mathrm{d} \phi^{2}\right),
$$

where $-\infty<w \leq u<\infty$. To employ Penrose's technique [12] of bringing infinity into finite distance, new null coordinates that assign finite values to the infinities of $u, w$ must be defined. A possible choice is $p=\arctan u$ and $q=\arctan w$ with $-\pi / 2<q \leq p<\pi / 2$. Notice that the infinities $\pm \infty$ of $u, w$ have been mapped to the finite values $\pm \pi / 2$ of $p, q$. In these coordinates the Minkowski metric takes the form

$$
\bar{g}_{M}=\Omega^{-2}\left[4 \mathrm{~d} p \mathrm{~d} q-\sin ^{2}(p-q)\left(\mathrm{d} \theta^{2}+\sin ^{2} \theta \mathrm{d} \phi^{2}\right)\right],
$$

where the conformal factor $\Omega(p, q)=2 \cos p \cos q$ is positive definite in the domain $p, q \in(-\pi / 2, \pi / 2)$ and has the appropriate behavior at infinity, i.e., $\Omega( \pm \pi / 2$, $\pm \pi / 2)=0$. Thus, the metric $\bar{g}_{M}$ has been conformally transformed to the metric

$$
g_{M}=4 \mathrm{~d} p \mathrm{~d} q-\sin ^{2}(p-q)\left(\mathrm{d} \theta^{2}+\sin ^{2} \theta \mathrm{d} \phi^{2}\right) .
$$

Finally, effecting the transformation $(T, R)=(p+q$, $p-q$ ), the conformal metric $g_{M}$ can be brought into the form (2.1), namely,

$$
g_{M}=\mathrm{d} T^{2}-\mathrm{d} R^{2}-\sin ^{2} R\left(\mathrm{~d} \theta^{2}+\sin ^{2} \theta \mathrm{d} \phi^{2}\right),
$$

where the coordinates now must satisfy the relations 


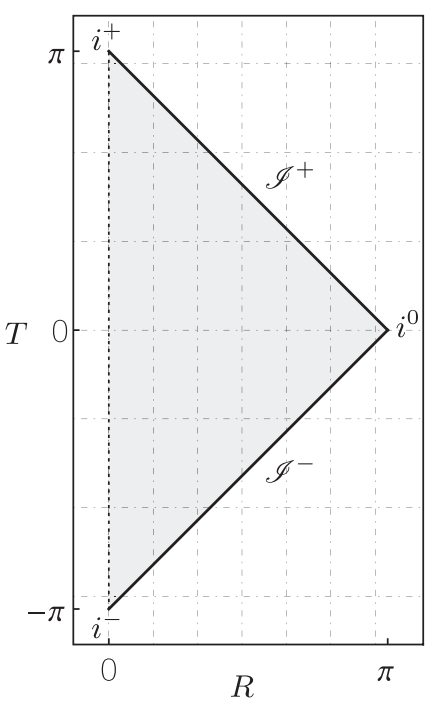

(a)

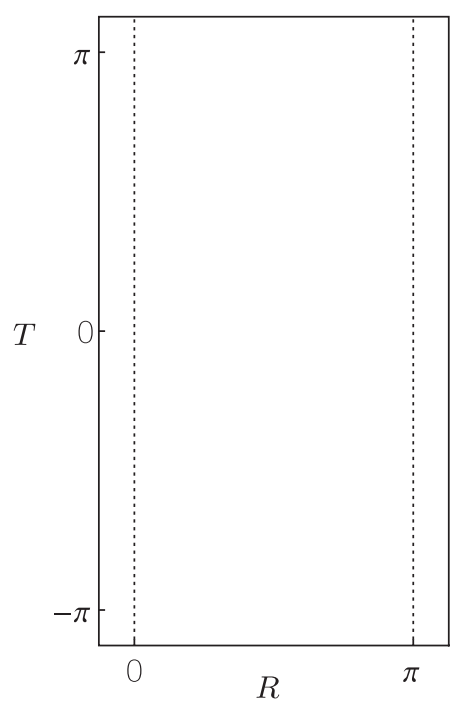

(b)

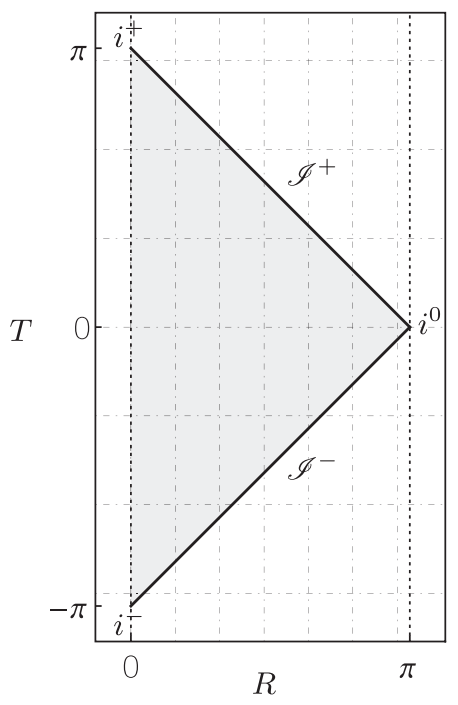

(c)

FIG. 1. Conformal compactification of Minkowski spacetime into the Einstein static universe. Conformal diagrams of (a) Minkowski, (b) the Einstein static universe (2.1) and (2.2), and (c) Minkowski embedded in the Einstein static universe (2.4) and (2.5) on the ( $T, R$ ) plane are shown. The usual rules apply: Every point in these diagrams represents a 2 -sphere, except for those on dotted lines, which represent points. Points on solid lines are points at infinity. The structure of conformal infinity is clearly visible.

$$
\begin{aligned}
-\pi & <T+R<\pi, \quad-\pi<T-R<\pi, \quad-\pi<T<\pi, \\
0 & <R<\pi, \quad 0<\theta<\pi, \quad-\pi<\phi<\pi .
\end{aligned}
$$

Conditions (2.5) restrict the whole of Minkowski spacetime $\mathbb{M}$ to a finite open subset of the Einstein static universe $\mathbb{E}$-compare with (2.1) and (2.2). In fact, the conformal infinity of Minkowski spacetime lies at the boundary of this region. The location of this boundary is given by the vanishing of the conformal factor $\Omega$, which in the coordinates $(T, R, \theta, \phi)$, reads

$$
\Omega(T, R)=2 \cos \left(\frac{T+R}{2}\right) \cos \left(\frac{T-R}{2}\right)
$$

Thus, the structure of conformal infinity is as follows (see Fig. 1). Past timelike infinity $i^{-}$, spacelike infinity $i^{0}$, and future timelike infinity $i^{+}$are located at the coordinate points $(T, R)=(-\pi, 0),(T, R)=(0, \pi)$, and $(T, R)=$ $(\pi, 0)$, respectively. Past null infinity $\mathcal{I}^{-}$and future null infinity $\mathcal{I}^{+}$are given by the hypersurfaces $T=R-\pi$ and $T=\pi-R$ with $0<R<\pi$, respectively.

\section{B. Blowing up of spacelike infinity to a cylinder}

Although the metric (2.4) extends smoothly to spacelike infinity, reconstructing the Minkowski spacetime described by it, from initial data that satisfy the conformal constraints, is not trivial at all, as some of the initial data exhibit a singular behavior at the point $i^{0}$. Following the discussion in [25], one can render the initial data regular by performing an appropriate rescaling of (2.4). In this new picture spacelike infinity $i^{0}$ has a finite representation as a cylinder (see Fig. 2). In the following we describe how to blow up the point $(T, R)=(0, \pi)$ that represents spacelike infinity $i^{0}$ on the Einstein static universe.

First, in accordance with [25], the spacetime metric (2.4) is rescaled to

$$
g=\kappa^{-2} g_{M}=\kappa^{-2}\left[\mathrm{~d} T^{2}-\mathrm{d} R^{2}-\sin ^{2} R\left(\mathrm{~d} \theta^{2}+\sin ^{2} \theta \mathrm{d} \phi^{2}\right)\right],
$$

and then new time $t$ and spatial $r$ coordinates are introduced by the transformation $(T, R)=(\kappa(r) f(t), \pi r)$ to get

$$
\begin{aligned}
g= & \dot{f}^{2} \mathrm{~d} t^{2}+\frac{2 \kappa^{\prime} f \dot{f}}{\kappa} \mathrm{d} t \mathrm{~d} r-\frac{\left(\pi^{2}-f^{2} \kappa^{\prime 2}\right)}{\kappa^{2}} \mathrm{~d} r^{2} \\
& -\frac{\sin ^{2}(\pi r)}{\kappa^{2}}\left(\mathrm{~d} \theta^{2}+\sin ^{2} \theta \mathrm{d} \phi^{2}\right),
\end{aligned}
$$

where 'and ' denote differentiation with respect to $t$ and $r$, respectively. Notice that (2.7) is spherically symmetric and that the new spatial coordinate is in the interval $0<r<1$. As we will see below, the functions $\kappa(r)$ and $f(t)$ control the shape and the location of the cylinder and of null infinity $\mathcal{I}^{ \pm}$with respect to the coordinates $t$ and $r$.

Specifically, as we will see in the next section, the rescaling function $\kappa$ multiplies the spatial derivatives in the evolution equations. Thus, according to [25], $\kappa$ must vanish on the cylinder, i.e., at $r=1$. In addition, $\kappa$ must be even with respect to $r=0$ to maintain a regular center. There is a plethora of functions that satisfy these criteria; in the following we will work with the choice 


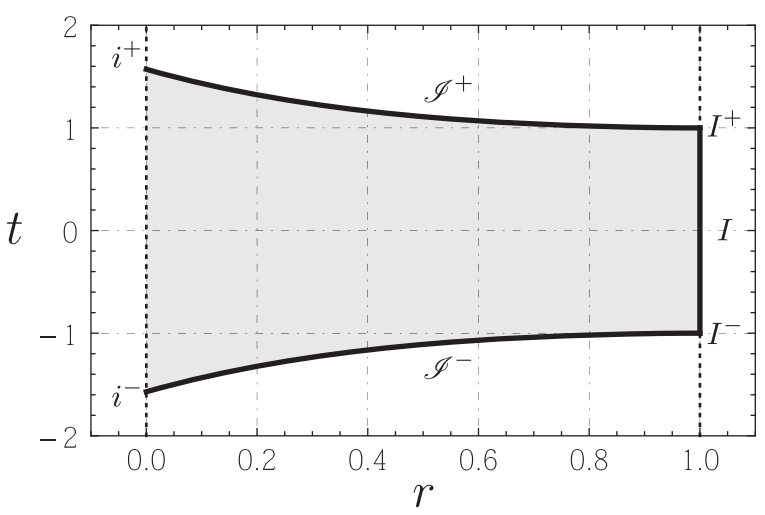

(a)

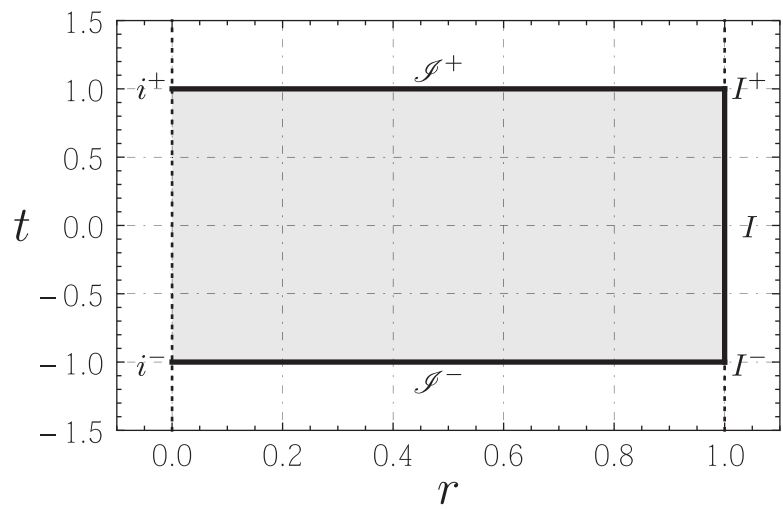

(b)

FIG. 2. Conformal compactification of Minkowski spacetime into the Einstein static universe with spacelike infinity $i^{0}$ blown up to a cylinder of finite size. The Einstein static universe $\mathbb{E}$ spans the area between the dotted vertical lines at $r=0$ and $r=1$. We give conformal diagrams of Minkowski spacetime embedded in the Einstein static universe (2.7) on the $(t, r)$ plane for the choices $\kappa(r)=$ $\cos \left(\frac{\pi r}{2}\right)$ and (a) $f(t)=2 t$ and (b) $f(t)=\frac{1}{20} \operatorname{arctanh}(t)$. The shaded areas denote the part of $\mathbb{E}$ that is conformal to Minkowski spacetime. The structure of conformal infinity for the (a) nonhorizontal (2.15) and (b) horizontal (2.17) cases is clearly visible.

$$
\kappa(r)=\cos \left(\frac{\pi r}{2}\right) .
$$

The time-dependent function $f$, on the other hand, appears in the coefficients of the time derivatives of the evolution equations; thus, it controls the size of the cylinder and the shape of null infinity $\mathcal{I}^{ \pm}$. It turns out that the vanishing of the overall conformal factor $\Theta=\kappa^{-1} \Omega$ between the metrics (2.3) and (2.7), i.e., $g=\Theta^{2} \bar{g}_{M}$, dictates the choice of $f(t)$. Observing (2.6) and (2.8), the conformal factor $\Theta$ in the new $(t, r)$ coordinates reads

$$
\Theta(t, r)=\frac{\Omega}{\kappa}=\frac{1}{\kappa}[\cos (\kappa f)+\cos (\pi r)] .
$$

As before, the vanishing of (2.9) locates the position of conformal infinity. Notice that now spacelike infinity is not represented as a point but as a cylinder of finite temporal extension with respect to the time coordinate $t$. For $0 \leq$ $r<1$ the condition $\Theta=0$ is equivalent to

$$
t= \pm f^{-1}\left(\frac{\pi(1-r)}{\kappa}\right)
$$

where $f^{-1}$ is the inverse of the time-dependent function $f(t)$. The position of $\mathcal{I}^{ \pm}$immediately follows,

$$
\mathcal{I}^{ \pm}=\left\{0 \leq r<1, t= \pm f^{-1}\left(\frac{\pi(1-r)}{\kappa}\right)\right\} .
$$

In addition, at $r=1$ the condition $\Theta=0$ is always satisfied, indicating the presence of the cylinder at this location. As expected, at the limit $r \rightarrow 1$, future and past null infinity do not meet at the same point as they do in the conventional picture (see Fig. 2). [Notice that (2.10) for $r \rightarrow 1$ gives $t= \pm f^{-1}(2)$.] Now null and spacelike infinity meet at the so-called critical sets

$$
I^{ \pm}=\left\{r=1, t= \pm f^{-1}(2)\right\}
$$

which are 2-spheres representing the bases of the cylinder. So the sets (2.12) are bounding from above and below the cylinder, and consequently, its position is given by the set

$$
I=\left\{r=1,-f^{-1}(2)<t<f^{-1}(2)\right\} .
$$

It is noteworthy that the height of the cylinder $I$ is $H_{I}=2 f^{-1}(2)$.

In the rest of the section, we will justify the choices of the time-dependent function $f$ that are going to be employed in the present work. One of the simplest possible choices for $f(t)$ is

$$
f(t)=2 t
$$

whose inverse is the function $f^{-1}(y)=y / 2$. Therefore, the structure of conformal infinity for the choice (2.14) follows from (2.11)-(2.13):

$$
\begin{aligned}
I & =\{r=1,-1<t<1\}, \\
I^{ \pm} & =\{r=1, t= \pm 1\}, \\
\mathcal{I}^{ \pm} & =\left\{0 \leq r<1, t= \pm \frac{\pi(1-r)}{2 \kappa}\right\} .
\end{aligned}
$$

A graphic representation of (2.15) is depicted in Fig. 2(a). If one wants to make $\mathcal{I}^{ \pm}$horizontal, then the function $f^{-1}$ in (2.10) must be chosen in such a way that it is constant in its domain of definition. A possible choice of $f$ that has this property is

$$
f(t)=\frac{1}{20} \operatorname{arctanh}(t) .
$$

Notice that the inverse of (2.16), i.e., $f^{-1}(y)=\tanh (20 y)$, is constant within machine accuracy in its domain 
$D_{f^{-1}}=[40,20 \pi]$. Specifically, the expression (2.10) for the choice (2.16) reads $t= \pm 1$, and consequently, conformal infinity [see Fig. 2(b)] has the following structure:

$$
\begin{aligned}
I & =\{r=1,-1<t<1\}, \\
I^{ \pm} & =\{r=1, t= \pm 1\}, \\
\mathcal{I}^{ \pm} & =\{0 \leq r<1, t= \pm 1\} .
\end{aligned}
$$

The main advantage of the horizontal representation (2.17) is that the whole of Minkowski spacetime has been mapped to a rectangle of finite size [see Fig. 2(b)]. As we will see in Sec. IV, this representation is numerically very advantageous.

\section{GENERALIZED CONFORMAL FIELD EQUATIONS}

Here the generalized conformal field equations [25] will be linearized on Minkowski spacetime. On a Minkowski background, such as the one described in the previous section, their linearization simplifies considerably. Namely, the so-called structural equations defining the torsion and the curvature are trivially satisfied [31,35]; thus, one is left with the remaining Bianchi identity for the perturbation of the rescaled Weyl tensor. In the present section the spinor version of this equation, i.e., the so-called spin- 2 zero-restmass equation [36], will be studied analytically as a system of first- and second-order partial differential equations (PDEs). Therefore, in the rest of this work the 2-spinor formalism [37] will be used. We employ this formalism as it simplifies our arguments and makes the manipulation of the quantities relevant to our study - which also emerge more naturally in this formalism-easier.

\section{A. Spin-2 zero-rest-mass equation}

Here we describe how to obtain a coordinate representation of the spin-2 zero-rest-mass equation as a system of first-order PDEs and thoroughly study its analytical properties.

\section{Basis, connections, and spin coefficients}

We start by defining a basis and a connection compatible with (2.7). To do so, we first introduce a spin frame $\left(o^{A}, t^{A}\right)$, with the usual normalization condition $o_{A} l^{A}=1$, and then form a coordinate basis by defining the nonorthonormal null tetrad $\left(l^{\mu}, n^{\mu}, m^{\mu}, \bar{m}^{\mu}\right)$ with

$$
\begin{aligned}
l^{\mu} & =\frac{1}{\sqrt{2}}(A, B, 0,0), \\
n^{\mu} & =\frac{1}{\sqrt{2}}(C,-B, 0,0), \\
m^{\mu} & =\frac{1}{\sqrt{2} g r}(0,0,1,-\mathrm{i} \csc \theta), \\
\bar{m}^{\mu} & =\frac{1}{\sqrt{2} g r}(0,0,1, \mathrm{i} \csc \theta),
\end{aligned}
$$

where the functions $A(t, r), B(t, r) C(t, r)$, and $g(r)$ are uniquely defined in terms of the metric coefficients of (2.7), i.e.,

$$
\begin{aligned}
A & =\frac{1}{\dot{f}}\left(1-\frac{\kappa^{\prime} f}{\pi}\right), \quad B=\frac{\kappa}{\pi}, \quad C=\frac{1}{\dot{f}}\left(1+\frac{\kappa^{\prime} f}{\pi}\right), \\
g & =\frac{\sin (\pi r)}{\kappa r} .
\end{aligned}
$$

It can be readily confirmed that the null vectors (3.1) satisfy the correct inner product conditions, $l^{\mu} n_{\mu}=-m^{\mu} \bar{m}_{\mu}=1$, and all the remaining combinations vanish.

The directional derivatives along the null vectors (3.1) immediately follow:

$$
\begin{aligned}
D & =l^{\mu} \partial_{\mu}=\frac{1}{\sqrt{2}}\left(A \partial_{t}+B \partial_{r}\right), \\
D^{\prime} & =n^{\mu} \partial_{\mu}=\frac{1}{\sqrt{2}}\left(C \partial_{t}-B \partial_{r}\right), \\
\delta & =m^{\mu} \partial_{\mu}=\frac{1}{\sqrt{2} g r}\left(\partial_{\theta}-\mathrm{i} \csc \theta \partial_{\phi}\right), \\
\delta^{\prime} & =\bar{m}^{\mu} \partial_{\mu}=\frac{1}{\sqrt{2} g r}\left(\partial_{\theta}+\mathrm{i} \csc \theta \partial_{\phi}\right),
\end{aligned}
$$

where $A, B, C, g$ are the same as above. The commutation relations among the directional derivatives (3.3) provide us with the spin coefficients corresponding to (2.7) and (3.1). The nonvanishing spin coefficients are listed below:

$$
\begin{aligned}
& \alpha=-\beta=\frac{\cot \theta}{2 \sqrt{2} g r}, \quad \gamma=\epsilon=-\frac{\kappa^{\prime}}{2 \sqrt{2} \pi}, \\
& \rho=-\rho^{\prime}=\frac{\kappa^{\prime}-\pi \kappa \cot (\pi r)}{\sqrt{2} \pi} .
\end{aligned}
$$

Next, we define the weighted differential operators of the Geroch-Held-Penrose (GHP) formalism in terms of the directional derivatives (3.3) and the spin coefficients (3.4):

$$
\begin{array}{ll}
\mathrm{P} \eta=(D-2 w \gamma) \eta, & \mathrm{P}^{\prime} \eta=\left(D^{\prime}-2 w \gamma\right) \eta, \\
\Varangle \eta=(\delta+2 s \alpha) \eta, & \partial^{\prime} \eta=\left(\delta^{\prime}-2 s \alpha\right) \eta,
\end{array}
$$

where $\eta$ is a $\{p, q\}$-scalar quantity with boost weight $w=\frac{p+q}{2}$ and spin weight $s=\frac{p-q}{2}$ (see [37]).

\section{Derivation}

Having defined a basis, derivatives, and spin coefficients compatible with the metric (2.7), we proceed further and express the spin-2 zero-rest-mass equation as a system of first-order PDEs. Our starting point is the spinor version of the Bianchi identity for the Weyl tensor on a general spacetime with metric $\tilde{g}$, which in the absence of matter reads 


$$
\tilde{\nabla}_{A^{\prime}}^{A} \tilde{\Psi}_{A B C D}=0,
$$

where $\tilde{\Psi}_{A B C D}$ is the spinor counterpart of the Weyl tensor for the metric $\tilde{g} ; \Psi_{A B C D}$ is called the Weyl spinor and is totally symmetric in its indices $\tilde{\Psi}_{A B C D}=\tilde{\Psi}_{(A B C D)}$; and $\tilde{\nabla}_{A A^{\prime}}$ is the spinor covariant derivative (see [37]). The generalized conformal field equations are concerned with the conformal structure of a spacetime, i.e., the structure that remains invariant under conformal rescalings of the metric $\tilde{g} \mapsto g=\Theta^{2} \tilde{g}$. Under these transformations the Weyl spinor remains invariant, $\Psi_{A B C D}=\tilde{\Psi}_{A B C D}$. The behavior of the spinor Bianchi identity (3.6) under this kind of conformal transformation reads [37]

$$
\nabla_{A^{\prime}}^{A}\left(\Theta^{-1} \Psi_{A B C D}\right)=\Theta^{-2} \tilde{\nabla}_{A^{\prime}}^{A} \tilde{\Psi}_{A B C D},
$$

which through (3.6) results in

$$
\nabla^{A}{ }_{A^{\prime}} \Delta_{A B C D}=0,
$$

the so-called Bianchi equation for the rescaled Weyl spinor $\Delta_{A B C D}=\Theta^{-1} \Psi_{A B C D}$.

Now, in order to obtain the weak field limit of (3.7), i.e., the equation for a small perturbation of a Minkowski spacetime, we consider, as in [37], that our spacetime metric is a smoothly varying function $g(u)$ of a single parameter $u$ such that $g(0)$ is conformal to the Minkowski spacetime. Similarly, one expects that the rescaled Weyl spinor $\Delta_{A B C D}(u)$ for any nonzero value of $u$ satisfies (3.7) and tends smoothly to zero as $u \rightarrow 0$. The latter guarantees that the totally symmetric spinor quantity $u^{-1} \Delta_{A B C D}(u)$ has a well-defined limit, say, $\Phi_{A B C D}$, as $u \rightarrow 0$ and satisfies the equation

$$
\nabla_{A^{\prime}}^{A} \Phi_{A B C D}=0
$$

where the totally symmetric spinor $\Phi_{A B C D}$ is called the spin-2 zero-rest-mass field. The spin-2 zero-rest-mass equation (3.8) is the spinor version of the Bianchi equation for the rescaled Weyl tensor.

\section{Coordinate representation}

In order to proceed further in our study of (3.8), we have to decompose it into its components. To do so, one first has to express the spinor covariant derivative in (3.8), as in [31], in terms of the weighted differential operators (3.5) of the GHP formalism:

$$
\nabla^{A}{ }_{A^{\prime}}=\imath^{A} l_{A^{\prime}} \mathrm{P}+o^{A} o_{A^{\prime}} \mathrm{P}^{\prime}-\imath^{A} o_{A^{\prime}} \circlearrowright-o^{A} l_{A^{\prime}} \nearrow^{\prime} ;
$$

we then substitute this expression in (3.8) and expand the spin-2 field in terms of its five independent components $\left(\Phi_{0}, \Phi_{1}, \Phi_{2}, \Phi_{3}, \Phi_{4}\right)$; finally, we take the components of the resulting expression to get

$$
\begin{aligned}
& \mathrm{P} \Phi_{k}-\nearrow^{\prime} \Phi_{k-1}=(5-k) \rho \Phi_{k}, \\
& \mathrm{P}^{\prime} \Phi_{k-1}-\partial \Phi_{k}=-k \rho \Phi_{k-1},
\end{aligned}
$$

where $k=1,2,3,4$ and $\rho$ is given by (3.4). Next, by using (3.5) and (3.3) it is possible to obtain a coordinate representation of the system (3.10),

$$
\begin{aligned}
& A \partial_{t} \Phi_{k}+B \partial_{r} \Phi_{k}-\sqrt{2}[(5-k) \rho+2(2-k) \epsilon] \Phi_{k}=\sqrt{2} \partial^{\prime} \Phi_{k-1}, \\
& C \partial_{t} \Phi_{k-1}-B \partial_{r} \Phi_{k-1}+\sqrt{2}[k \rho-2(3-k) \epsilon] \Phi_{k-1}=\sqrt{2} \partial \Phi_{k},
\end{aligned}
$$

where $k=1,2,3,4$, the functions $A, B, C$ are given by (3.2), and the spin coefficients $\rho, \epsilon$ by (3.4). Observing (3.3)-(3.5), the $\partial, \partial^{\prime}$ operators can be expressed on the unit sphere through the transition

$$
\partial \mapsto \frac{1}{\sqrt{2} g r} \partial_{0} \quad \text { and } \quad \partial^{\prime} \mapsto \frac{1}{\sqrt{2} g r} \partial_{0}^{\prime},
$$

where $\partial_{0}=\partial_{\theta}-\mathrm{i} \csc \theta \partial_{\phi}+s \cot \theta \quad$ and $\quad \partial_{0}^{\prime}=\partial_{\theta}+$ $i \csc \theta \partial_{\phi}-s \cot \theta$ denote the "eth" operators on the unit sphere. Finally, we use the spherical symmetry of the metric (2.7) to expand the components $\phi_{k}$ of the spin-2 field as a sum of spin-weighted spherical harmonics ${ }_{s} Y_{l m}$ in the following way:

$$
\phi_{k}(t, r, \theta, \phi)=\sum_{l m} \phi_{k}^{l m}(t, r)_{2-k} Y_{l m}(\theta, \phi),
$$

where $s=2-k$ is the spin weight of $\phi_{k}$ and the integers $s$, $l, m$ satisfy the inequalities $|s| \leq l$ and $|m| \leq l$. Since the operators $\partial_{0}, \partial_{0}^{\prime}$ act on the spin-weighted spherical harmonics ${ }_{s} Y_{l m}$ as

$$
\begin{aligned}
\partial_{0}\left({ }_{s} Y_{l m}\right) & =-\sqrt{l(l+1)-s(s+1)}_{s+1} Y_{l m}, \\
\partial_{0}{ }^{\prime}\left({ }_{s} Y_{l m}\right) & =\sqrt{l(l+1)-s(s-1)}{ }_{s-1} Y_{l m},
\end{aligned}
$$

the system (3.11) decouples into separate systems for each mode of the fixed pair $(l, m)$, i.e.,

$$
\begin{aligned}
& A \partial_{t} \Phi_{k}+B \partial_{r} \Phi_{k}-\sqrt{2}[(5-k) \rho+2(2-k) \epsilon] \Phi_{k} \\
& \quad=\frac{\alpha_{(2-k)(3-k)}}{g r} \Phi_{k-1}, \\
& C \partial_{t} \Phi_{k-1}-B \partial_{r} \Phi_{k-1}+\sqrt{2}[k \rho-2(3-k) \epsilon] \Phi_{k-1} \\
& \quad=-\frac{\alpha_{(2-k)(3-k)}}{g r} \Phi_{k},
\end{aligned}
$$

where $k=1,2,3,4, \alpha_{n} \equiv \sqrt{l(l+1)-n}$, the functions $A$, $B, C, g$ are given by (3.2), and the spin coefficients $\rho, \epsilon$ by (3.4). The eight equations (3.15), for the five independent components $\Phi_{k}$ of the spin-2 field, comprise a coordinate representation of (3.8) on the background spacetime (2.7). 


\section{Evolution and constraint equations}

The system (3.15) can be readily split into five evolution equations,

$$
\begin{aligned}
C \partial_{t} \Phi_{0} & =B \partial_{r} \Phi_{0}+4 \sqrt{2} \epsilon \Phi_{0}-\sqrt{2} \rho \Phi_{0}-\frac{\alpha_{2}}{g r} \Phi_{1}, \\
(A+C) \partial_{t} \Phi_{1} & =4 \sqrt{2} \epsilon \Phi_{1}+2 \sqrt{2} \rho \Phi_{1}+\frac{\alpha_{2}}{g r} \Phi_{0}-\frac{\alpha_{0}}{g r} \Phi_{2}, \\
(A+C) \partial_{t} \Phi_{2} & =\frac{\alpha_{0}}{g r} \Phi_{1}-\frac{\alpha_{0}}{g r} \Phi_{3}, \\
(A+C) \partial_{t} \Phi_{3} & =-4 \sqrt{2} \epsilon \Phi_{3}-2 \sqrt{2} \rho \Phi_{3}+\frac{\alpha_{0}}{g r} \Phi_{2}-\frac{\alpha_{2}}{g r} \Phi_{4}, \\
A \partial_{t} \Phi_{4} & =-B \partial_{r} \Phi_{4}-4 \sqrt{2} \epsilon \Phi_{4}+\sqrt{2} \rho \Phi_{4}+\frac{\alpha_{2}}{g r} \Phi_{3},
\end{aligned}
$$

and three constraints,

$$
\begin{aligned}
C_{1} \equiv & 2(A+C) B \partial_{r} \Phi_{1}+4 \sqrt{2} A(\epsilon-\rho) \Phi_{1} \\
& -4 \sqrt{2} C(2 \rho+\epsilon) \Phi_{1}-\frac{2 \alpha_{0}}{g r} A \Phi_{2}-\frac{2 \alpha_{2}}{g r} C \Phi_{0}=0, \\
C_{2} \equiv & 2(A+C) B \partial_{r} \Phi_{2}-6 \sqrt{2}(A+C) \rho \Phi_{2}-\frac{2 \alpha_{0}}{g r} C \Phi_{1} \\
& -\frac{2 \alpha_{0}}{g r} A \Phi_{3}=0, \\
C_{3} \equiv & 2(A+C) B \partial_{r} \Phi_{3}+4 \sqrt{2} C(\epsilon-\rho) \Phi_{3} \\
& -4 \sqrt{2} A(2 \rho+\epsilon) \Phi_{3}-\frac{2 \alpha_{0}}{g r} C \Phi_{2} \\
& -\frac{2 \alpha_{2}}{g r} A \Phi_{4}=0 .
\end{aligned}
$$

Notice that the evolution system (3.16) acquires a very simple form on the cylinder $I$ (i.e., at $r=1$ ). The radial derivatives are multiplied by the function $B$ defined in (3.2), which for the choice (2.8) vanishes at $r=1$. Thus, the radial derivatives drop out from the equations controlling the dynamics of the components $\Phi_{0}, \Phi_{4}$, and the cylinder $I$ becomes a total characteristic of the system (3.16). This feature, together with the fact that $I$, as can be seen in Fig. 2, lies on the boundary located at $r=1$ of the computational domain and indicates that we are not allowed to impose boundary conditions at points lying on $I$.

To check the well-posedness of the Cauchy problem described by the system (3.16) and (3.17), one has to write the evolution equations in a matrix form for the vector $\Phi \equiv\left(\Phi_{0}, \Phi_{1}, \Phi_{2}, \Phi_{3}, \Phi_{4}\right)^{\mathrm{T}}$ :

$$
\mathbf{A}_{0} \partial_{t} \Phi+\mathbf{A}_{1} \partial_{r} \Phi=\mathbf{A}_{2} \Phi
$$

where $\mathbf{A}_{0}=\operatorname{diag}(A, A+C, A+C, A+C, C)$ and $\mathbf{A}_{1}=$ $\operatorname{diag}(-B, 0,0,0, B)$ are obviously Hermitian matrices, and
$\mathbf{A}^{0}$ is positive definite when the conditions $\dot{f}(t)>0$ and $|f|<\pi /\left|\kappa^{\prime}\right|$ hold simultaneously. Therefore, the evolution system is symmetric hyperbolic, and consequently well posed, in the range $|t|<f^{-1}\left(\pi /\left|\kappa^{\prime}\right|\right)$-given that the condition $\dot{f}>0$ is also satisfied in this range.

To prove that the constraints (3.17) are preserved during the evolution, one has to derive the subsidiary system for the constraint quantities $C_{k}$ appearing in (3.17):

$$
\begin{aligned}
& \partial_{t} C_{1}=-\kappa \dot{f} \cot (\pi r) C_{1}-\frac{1}{2} \alpha_{0} \kappa \dot{f} \csc (\pi r) C_{2}, \\
& \partial_{t} C_{2}=\frac{1}{2} \alpha_{0} \kappa \dot{f} \csc (\pi r) C_{1}-\frac{1}{2} \alpha_{0} \kappa \dot{f} \csc (\pi r) C_{3}, \\
& \partial_{t} C_{3}=\frac{1}{2} \alpha_{0} \kappa \dot{f} \csc (\pi r) C_{2}+\kappa \dot{f} \cot (\pi r) C_{3},
\end{aligned}
$$

where, for the sake of simplicity, the functions $\kappa, f$ were used here instead of the $A, B, C, g$ functions and the spin coefficients $\rho, \epsilon$. The above system can be written in the symmetric hyperbolic form $\partial_{t} \mathbf{C}=\mathbf{A}_{3} \mathbf{C}$ for the vector $\mathbf{C} \equiv\left(C_{1}, C_{2}, C_{3}\right)^{\mathrm{T}}$, where the entries of the $3 \times 3$ matrix $\mathbf{A}_{3}$ consist of the coefficients of the rhs of the above subsidiary system. The eigenvalues of $\mathbf{A}_{3}$ read $\lambda=0, \pm \mathrm{i} \csc (\pi r) \kappa \dot{f} \sqrt{\alpha_{0}^{2}-2 \cos ^{2}(\pi r)} / \sqrt{2}$. The imaginary nature of the nontrivial eigenvalues guarantees that the unavoidable initial violation of the constraints does not grow exponentially during the evolution.

\section{Characteristic curves}

It is extremely useful to study the behavior of the characteristic curves of the evolution system (3.16) as their form provides a qualitative insight on the behavior of the solutions of (3.16). For first-order PDEs with a principal part of the form $a(t, r) \partial_{t} u(t, r)+b(t, r) \partial_{r} u(t, r)$, like the ones in (3.16), the slope of the characteristics is given by $\mathrm{d} t / \mathrm{d} r=a / b$. For the components $\Phi_{1}, \Phi_{2}, \Phi_{3}$ of the spin-2 field, the characteristics are straight lines of constant $r$ as $b$ vanishes in the corresponding evolution equations. The slopes of the remaining two components $\Phi_{0}$ and $\Phi_{4}$ read

$$
\frac{\mathrm{d} t}{\mathrm{~d} r}=-\frac{C}{B} \quad \text { and } \quad \frac{\mathrm{d} t}{\mathrm{~d} r}=\frac{A}{B},
$$

respectively. As expected, the behavior of the above characteristics depends entirely on the choice of the functions $\kappa$ and $f$ [see (3.2)]. Figure 3 depicts the form of the characteristic curves for $\Phi_{0}$ and $\Phi_{4}$. Therein, the characteristics for the choices (2.14) and (2.16) of the timedependent function $f$ are presented.

Specifically, Fig. 3(a) depicts the characteristic curves of $\Phi_{0}$ (blue solid lines) and $\Phi_{4}$ (red dashed lines) for the choice (2.14). Notice that $\mathcal{I}^{+}$(upper black thick solid line) confines the characteristics of $\Phi_{0}$ from above, while $\mathcal{I}^{-}$ (lower black thick solid line) bounds the characteristics of 


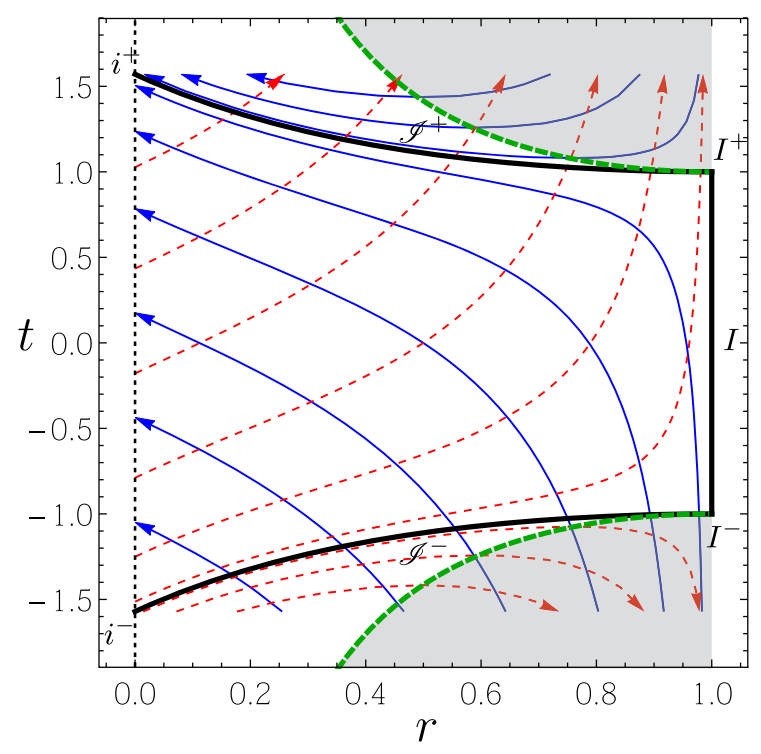

(a)

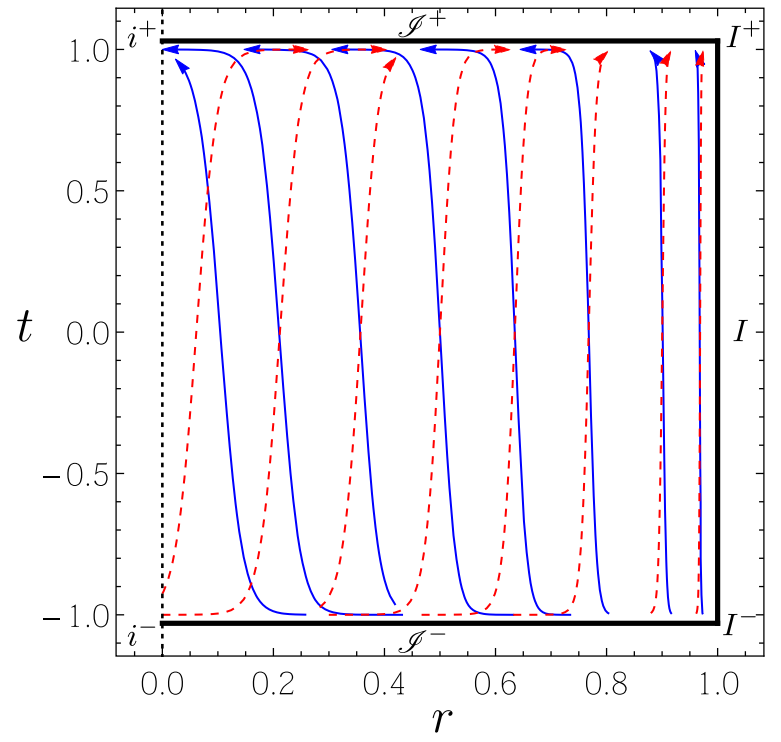

(b)

FIG. 3. Characteristic curves of the fields $\Phi_{0}$ (blue solid lines) and $\Phi_{4}$ (red dashed lines). The characteristic curves for the choices (a) $f(t)=2 t$ and (b) $f(t)=\frac{1}{20} \operatorname{arctanh}(t)$ are presented. The black thick solid lines represent conformal infinity, and the green thick dashed lines represent the span of the symmetric hyperbolic region. The shaded areas mark the domain of nonhyperbolicity of (3.16). In (b) conformal infinity and the boundary of the symmetric hyperbolic region coincide; thus, the system (3.16) is not symmetric hyperbolic in the region exterior to conformal infinity (this area has not been shaded for presentational reasons).

$\Phi_{4}$ from below. This observation is related to the wellknown fact [25] that the evolution equations of $\Phi_{0}$ and $\Phi_{4}$ degenerate at the corresponding critical sets $I^{+}$and $I^{-}$. The reason for this expected behavior of the evolution equations is clearly visible in Fig. 3(a). Namely, by trying to go beyond the critical sets $I^{ \pm}$, one enters the domain of nonhyperbolicity of the equations: shaded areas bounded by the green thick dashed lines. In the remaining nonshaded area the system (3.16) is symmetric hyperbolic - this is the domain for which the condition of symmetric hyperbolicity $|t|<f^{-1}\left(\pi /\left|\kappa^{\prime}\right|\right)$ is satisfied. Therefore, the whole of the conformally compactified Minkowski spacetime [see shaded region of Fig. 2(a) and compare with Fig. 3(a)] is situated entirely in the symmetric hyperbolic region of (3.16). As was also mentioned above, the shape of the characteristic curves clearly shows that the cylinder $I$ is a total characteristic of our system.

The situation is quite similar for the choice (2.16) illustrated in Fig. 3(b). The parts of $\mathbb{E}$ that are conformal to Minkowski spacetime are located entirely inside the domain $|t|<1$ of symmetric hyperbolicity, which now coincides with the interior of the black thick solid lines representing conformal infinity. Thus, there is no reason for going beyond the critical sets $I^{ \pm}$and entering the region of nonhyperbolicity, as now by reaching $I^{ \pm}$, one actually reaches timelike infinity $i^{ \pm}$. The behavior of the characteristics here is more universal in the sense that the characteristics for both fields $\Phi_{0}$ and $\Phi_{4}$ are bounded from above and below by null infinity. Thus, the evolution equations for $\Phi_{0}$ and $\Phi_{4}$ degenerate at both critical sets $I^{ \pm}$.

\section{B. Spin-2 zero-rest-mass wave equation}

In the current section, we present a coordinate representation of the spin-2 zero-rest-mass equation as a system of second-order PDEs and discuss its analytical properties.

\section{Derivation}

Our starting point is the spin-2 zero-rest-mass equation (3.8). We apply the spinor covariant derivative $\nabla_{A}^{A^{\prime}}$ to it and contract over the $A^{\prime}$ index to get

$$
\nabla_{A^{\prime} A} \nabla_{F}^{A^{\prime}} \Phi_{B C D}^{F}=0,
$$

where the indices have been moved appropriately in order to be able to split the above differential operator into its symmetric and skew symmetric parts in $A F$,

$$
\nabla_{A^{\prime} A} \nabla_{F}^{A^{\prime}} \Phi_{B C D}^{F}=\square_{A F} \Phi_{B C D}^{F}-\frac{1}{2} \square \Phi_{A B C D}=0,
$$

where $\square_{A F} \equiv \nabla_{A^{\prime}(A} \nabla_{F)}^{A^{\prime}}$ and $\square \equiv \nabla_{A A^{\prime}} \nabla^{A A^{\prime}}$. Next, using the identities

$$
\begin{aligned}
& \square_{A F} k^{C}=\left[\Psi_{A F E}^{C}+\frac{R}{24}\left(\epsilon_{A E} \epsilon_{F}^{C}+\epsilon_{F E} \epsilon_{A}^{C}\right)\right] k^{E}, \\
& \square_{A F} k_{C}=-\left[\Psi_{A F C}{ }^{E}+\frac{R}{24}\left(\epsilon_{A C} \epsilon_{F}^{E}+\epsilon_{F C} \epsilon_{A}{ }^{E}\right)\right] k_{E},
\end{aligned}
$$

where $R$ is the scalar curvature and $\Psi_{A B C D}$ is the Weyl spinor of (2.7), the above expression simplifies considerably, 


$$
-\frac{R}{4} \Phi_{A B C D}+3 \Psi_{E F(A B} \Phi_{C D)} E F-\frac{1}{2} \square \Phi_{A B C D}=0 .
$$

Now, taking into consideration that for the metric (2.7) the Weyl spinor vanishes and the scalar curvature reads

$$
R=\frac{3}{2} \kappa^{2},
$$

we finally arrive at the so-called spin- 2 zero-rest-mass wave equation

$$
\square \Phi_{A B C D}+\frac{3 \kappa^{2}}{4} \Phi_{A B C D}=0,
$$

which will be used to describe (3.8) as a system of secondorder PDEs.

\section{Coordinate representation}

Now we obtain a coordinate representation of (3.19). As before, we express the differential operator $\square$ in terms of the weighted differential operators (3.5) of the GHP formalism:

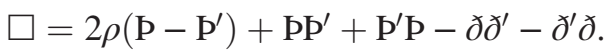

Substitute this expression into (3.19), expand the spin-2 field in terms of its components, and take the components of the resulting expression to obtain the system of five equations,

$$
\begin{aligned}
\mathrm{PD}^{\prime} & \Phi_{\lambda}+\mathrm{P}^{\prime} \mathrm{P} \Phi_{\lambda}+2 \rho\left(\mathrm{P}-\mathrm{P}^{\prime}\right) \Phi_{\lambda}-2\left(\lambda^{2}-4 \lambda-2\right) \rho^{2} \Phi_{\lambda} \\
& +\frac{3}{4} \kappa^{2} \Phi_{\lambda} \\
= & \partial \partial^{\prime} \Phi_{\lambda}+\partial^{\prime} \partial \Phi_{\lambda}+2(4-\lambda) \rho \partial \Phi_{\lambda+1}-\lambda \rho \partial^{\prime} \Phi_{\lambda-1},
\end{aligned}
$$

where $\lambda=0,1,2,3,4, \kappa$ is the rescaling function (2.8) and $\rho$ is given by (3.4). In a similar fashion as in Sec. III A 2 and [31], one can use the map (3.12), the expansion (3.13), and the identities (3.14) to decouple (3.20) on the unit sphere into separate systems for each admissible pair $(l, m)$. Then, by using (3.5) and (3.3), a coordinate representation of (3.20) can be obtained:

$$
\begin{aligned}
& A C \partial_{t t} \Phi_{\lambda}+B(C-A) \partial_{t r} \Phi_{\lambda}-B^{2} \partial_{r r} \Phi_{\lambda}+B\left(2 \sqrt{2} \rho-2 \sqrt{2} \epsilon-B^{\prime}\right) \partial_{r} \Phi_{\lambda} \\
& \quad+\frac{1}{2}\left(B\left(C^{\prime}-A^{\prime}\right)+2 \sqrt{2}[(A-C) \rho+(2 \lambda-5) A \epsilon+(2 \lambda-3) C \epsilon]+\dot{A} C+A \dot{C}\right) \partial_{t} \Phi_{\lambda} \\
& +\frac{3 \kappa^{2}}{4} \Phi_{\lambda}-2\left(\lambda^{2}-4 \lambda-2\right) \rho^{2} \Phi_{\lambda}+8(\lambda-2)^{2} \epsilon^{2} \Phi_{\lambda}=-\frac{\alpha_{(\lambda-1)(\lambda-2)}^{2}+\alpha_{(\lambda-2)(\lambda-3)}^{2}}{2 g^{2} r^{2}} \Phi_{\lambda} \\
& \quad-\frac{\sqrt{2}(4-\lambda) \alpha_{(\lambda-1)(\lambda-2)} \rho}{g r} \Phi_{\lambda+1}-\frac{\sqrt{2} \lambda \alpha_{(\lambda-2)(\lambda-3)} \rho}{g r} \Phi_{\lambda-1},
\end{aligned}
$$

where $\lambda=0,1,2,3,4$, the functions $A, B, C, g$ are given by (3.2), and the expansion coefficients $\rho, \epsilon$ by (3.4). The above system of five equations is the coordinate representation of the spin-2 zero-rest-mass wave equation (3.19) on a background of the form (2.7). Notice that, as in the case of (3.16), all the radial derivatives drop out from the above system when the equations are restricted to the cylinder $I$ at $r=1$. Thus, the cylinder is again a total characteristic of (3.21).

To classify the system of second-order PDEs (3.21), we have to look at its symbol. The associated symmetric matrix of the principal part of the symbol of (3.21) reads

$$
\left(\begin{array}{cc}
A C & \frac{1}{2} B(C-A) \\
\frac{1}{2} B(C-A) & -B^{2}
\end{array}\right)
$$

with eigenvalues

$$
\begin{aligned}
\lambda_{ \pm} & =\frac{1}{2}\left(\omega \pm \sqrt{\omega^{2}+\varepsilon^{2}}\right) \quad \text { where } \omega=A C-B^{2} \quad \text { and } \\
\varepsilon & =B(A+C) .
\end{aligned}
$$

The system (3.21) is symmetric hyperbolic whenever the above eigenvalues have opposite signs. Obviously, $\lambda_{+}$is always positive, while $\lambda_{-}$is always negative. So the only case where the system (3.21) fails to be symmetric hyperbolic is when at least one of the eigenvalues vanishes. For $\lambda_{-}$that happens when $\varepsilon=0$, while $\lambda_{+}$vanishes when $\varepsilon=0$ and $\omega=0$. The hyperbolicity of the system (3.21) for the choice (2.16) breaks down when $t= \pm 1$ and $r=1$, i.e., when $\varepsilon=0$ and consequently $\lambda_{-}=0$. Thus, in this case, the system (3.21) is symmetric hyperbolic in the rectangular $\{0<r<1,|t|<1\}$. [Recall that the domain of symmetric hyperbolicity of the first-order system (3.16) for the choice (2.16) is exactly the same.] For the choice (2.14), the condition $\varepsilon=0$ entails that the hyperbolicity of (3.21) breaks down ar $r=1$. In addition, $\omega=0$ leads to $t= \pm \sqrt{\pi^{2}-\kappa^{2}} /\left|\kappa^{\prime}\right|$. Combining the last two results, one concludes that the symmetric hyperbolicity of the system (3.21) for the choice (2.14) is guaranteed in the domain $\left\{0<r<1,|t|<\sqrt{\pi^{2}-\kappa^{2}} /\left|\kappa^{\prime}\right|\right\}$. Notice that the domain of hyperbolicity of (3.21) is now slightly larger than the one of the spin-2 zero-rest-mass equation (3.16). But, close to 
the cylinder $I$ at $r=1$, their behavior coincides as the rescaling function (2.8) tends to zero there.

In order to prescribe initial data and constrain the evolved data on each time slice of constant $t$, we supplement the five evolution equations (3.21) with the constraints (3.17) of the first-order system of PDEs. The details of this procedure will be discussed in Sec. III C.

\section{Characteristic curves}

Because of its hyperbolic nature, the system (3.21) has two real characteristic curves. The slope of the characteristic curves for second-order partial differential equations like (3.21) with the principal part of the form $a(t, r) \partial_{t t} u(t, r)+b(t, r) \partial_{t r} u(t, r)+c(t, r) \partial_{r r} u(t, r)$ reads $\mathrm{d} t / \mathrm{d} r=\left(b \pm \sqrt{b^{2}-4 a c}\right) / 2 c$. Substituting $a, b, c$ according to (3.21), the slope of the characteristics follows,

$$
\frac{\mathrm{d} t}{\mathrm{~d} r}=-\frac{C}{B} \quad \text { and } \quad \frac{\mathrm{d} t}{\mathrm{~d} r}=\frac{A}{B} .
$$

Interestingly, the nonlinear characteristics of the spin-2 zero-rest-mass equation are identical to those of the spin-2 zero-rest-mass wave equation. Thus, Fig. 3 can be used to visualise them. Figure 3 must be read with care though because of a small but substantial difference in the behavior of the characteristics. Now, their behavior is more universal in the sense that the characteristics of all the components of the spin-2 field, and not only of some specific components like in the case of the spin-2 zero-rest-mass equation, behave in the way depicted by Fig. 3. Therefore, the (solid) blue and (dashed) red lines are not characteristics of different field components but of all components of the spin-2 field simultaneously. In addition, the characteristics of all five independent components of the spin-2 field exhibit the behavior illustrated in Fig. 3. One also has to keep in mind that, as mentioned above, the symmetric hyperbolic region is now a little bit larger; i.e., the shaded area is a little bit smaller, but close to the critical sets $I^{ \pm}$, it coincides with the one represented by the green thick dashed lines of Fig. 3. Thus, as in the case of the spin-2 zero-rest-mass equation, the entire compactified Minkowski spacetime lies wholly in the symmetric hyperbolic region of (3.21). The remaining features of Fig. 3 also apply, as they are, to the second-order system of PDEs (3.21).

\section{Relating the first- and second-order PDE systems}

As the solution space of the second-order PDE system (3.19) is larger than that of the first-order system (3.8), it would be highly desirable if one could know under which conditions the solutions of the two systems are the same. In order to do this, we have to somehow establish a correspondence between the two systems that would unveil these conditions.
Following [28,31], we first define the spinor

$$
\Sigma_{A^{\prime} B C D} \equiv \nabla_{A^{\prime}}^{F} \phi_{F B C D},
$$

which is nothing other than the lhs of (3.8); we then act upon it with another spinor covariant derivative to obtain

$$
\nabla_{A A^{\prime}} \Sigma_{B C D}^{A^{\prime}}=\nabla_{A A^{\prime}} \nabla^{F A^{\prime}} \phi_{F B C D}
$$

In accordance with the discussion in Sec. III B 1, the rhs of the above expression is given by the lhs of (3.19); therefore,

$$
\nabla_{A A^{\prime}} \Sigma_{B C D}^{A^{\prime}}=\square \Phi_{A B C D}+\frac{3 \kappa^{2}}{4} \Phi_{A B C D}
$$

Now, assuming that $\Phi_{A B C D}$ is a solution of (3.8), the spinor (3.22) vanishes, and thus (3.23) reduces to (3.19); i.e., $\Phi_{A B C D}$ is also a solution of the spin-2 zero-rest-mass wave equation (3.19).

Conversely, assuming that the spin-2 field $\Phi_{A B C D}$ is a solution of (3.19), the expression (3.23) reduces to

$$
\nabla_{A A^{\prime}} \Sigma_{B C D}^{A^{\prime}}=0
$$

The above system can guarantee that $\Phi_{A B C D}$ is also a solution of (3.8), i.e., $\Sigma_{A^{\prime} B C D}=0$, in the case that (3.8) holds initially and (3.24) is well posed. As the condition $\left.\Sigma_{A^{\prime} B C D}\right|_{S}=0$ can always be satisfied on an initial hypersurface $S$, one just has to prove that the system (3.24) is well posed in order for $\Sigma_{A^{\prime} B C D}=0$ to hold throughout the evolution. To do so, we have to look at the symbol of the first-order differential expression (3.24). Taking into account the fact that the components of (3.9) are $\nabla_{A A^{\prime}}=$ $\left(\nabla_{00^{\prime}}=\mathrm{P}, \nabla_{01^{\prime}}=ð, \nabla_{10^{\prime}}={\nearrow^{\prime}}^{\prime}, \nabla_{11^{\prime}}=\mathrm{P}^{\prime}\right)$, the expansion (3.13) of the components of $\Sigma_{A^{\prime} B C D}$ in terms of the spinweighted spherical harmonics, and the relation (3.3)-(3.5) between the remaining GHP operators $\mathrm{P}, \mathrm{P}^{\prime}$ and the coordinate derivatives $\partial_{t}, \partial_{r}$, one can write the principal part of the symbol of (3.24) as follows:

$$
L^{p}=\sum_{\alpha=0}^{1} \Sigma_{\alpha}(t, r) D^{\alpha} \quad \text { with } \quad D^{\alpha}=\left(\partial_{t}, \partial_{r}\right),
$$

where $\Sigma_{\alpha}$ are $N \times N$ square matrices, with $N=8$ being the number of independent components of the spinor $\Sigma_{A^{\prime} B C D}$. Due to the fact that the first-order system of PDEs (3.24) is symmetric under the spinor prime operation [37], each of the GHP operators $\mathrm{P}, \mathrm{P}^{\prime}$ will act on an equal number of components of $\Sigma_{A^{\prime} B C D}$, i.e., $N / 2$. Therefore, the $\Sigma$ matrices read

$$
\begin{aligned}
& \Sigma_{0}=\frac{1}{\sqrt{2}} \operatorname{diag}(\underbrace{A, \ldots, A}_{N / 2}, \underbrace{C, \ldots, C}_{N / 2}), \\
& \Sigma_{1}=\frac{B}{\sqrt{2}} \operatorname{diag}(\underbrace{1, \ldots, 1}_{N / 2}, \underbrace{-1, \ldots,-1}_{N / 2}),
\end{aligned}
$$


where $A, B, C$ are given by (3.2). As the $\Sigma$ matrices are obviously Hermitian and $\Sigma_{0}$ is positive definite in the range $|t|<f^{-1}\left(\pi /\left|\kappa^{\prime}\right|\right)$ with $\dot{f}>0$, the system (3.24) is symmetric hyperbolic in this range and defines a well-posed problem for $\Sigma_{A^{\prime} B C D}$.

In summary, we proved that the spin-2 field is a solution of both (3.8) and (3.19) if and only if the initial data for both systems satisfy the constraints (3.17) of the first-order system (3.8). Therefore, in the numerical implementation of (3.19), the initial data will be determined by (3.17) and subsequently evolved with (3.21). In this way we ensure that the obtained numerical solutions are also solutions of (3.8).

\section{NUMERICAL IMPLEMENTATION AND RESULTS}

Here we give a detailed description of the numerical setting that will be used to numerically study the systems (3.16)-(3.17) and (3.21). In the present work we mainly focus on the second-order PDE system (3.21) as its numerical solutions have better properties than those of the corresponding first-order system (see, e.g., Ref. [33]). According to [33], numerical approximations based on second-order PDEs lead to better accuracy than the ones based on first-order PDEs and, in addition, they prevent the occurrence of spurious high-frequency waves traveling against the characteristics. As these claims were also confirmed numerically in [28,31], in Sec. IV D we only present our findings concerning (3.21).

\section{A. Numerical preliminaries}

The method of lines will be used to discretize the $1+1$ system (3.21). Accordingly, the PDE system (3.21) is reduced to a system of ordinary differential equations by discretizing the spatial coordinate $r$ with finite difference techniques. In accordance with the setting of Sec. II B, our computational domain is $D=[0,1]$. To obtain a finite representation of $D$, an equidistant grid $r_{i}=i h$ of grid spacing $h$ is introduced, where $i=0, \ldots, N, r_{N}=1$, and thus $h=1 / N$. The spin-2 field is discretized in a similar way, $\left(\Phi_{\lambda}\right)_{i}=\Phi_{\lambda}\left(r_{i}\right)$. Next, we have to approximate the spatial derivatives with appropriate finite difference operators. We choose to use fourth-order central difference operators to approximate the first and second derivatives appearing in (3.21) on the entire computational domain $D$ except for the grid points lying in the vicinity of the cylinder $I$ at $r=1$. There, we use one-sided summationby-parts finite difference operators as in [27,28,31]. (Therein, a lengthy discussion about the advantages of using the summation-by-parts operators can be found.) The reasons for this "inconsistency" will become apparent in Sec. IV B and are related to difficulties in the numerical implementation of the system (3.21) at the origin $r=0$, where some terms of (3.21) become singular.
Now one has to decide how to solve the resulting semidiscrete system of ordinary differential equations. In order to implement the system (3.21) numerically, we reduce it to a system that is first order in time and second order in space by introducing the first derivatives $\Psi_{\lambda}=$ $\partial_{t} \Phi_{\lambda}$ of the spin- 2 field $\Phi_{\lambda}$ as additional variables. Then the reduced system can be evolved in time with standard explicit fourth-order Runge-Kutta schemes. When higher accuracy is required, especially in studies near the region $\mathrm{I}^{+}$like in the case depicted in Fig. 3(b), time-step adaptive Runge-Kutta schemes that adapt the time step to the speed of the characteristic curves will be employed. The adaptive time step allows us to approach $I^{+}$arbitrarily closely, but not exactly, as the time step then becomes arbitrarily small.

A point that usually needs special attention is the imposition of boundary conditions at the boundaries of the computational domain. The mathematical framework developed in the previous sections makes the treatment of the boundaries a little bit easier. Specifically, as already mentioned in Sec. III A 4, the cylinder is a total characteristic of our system. Therefore, we are not allowed to prescribe boundary conditions at the points that lie there. At the boundary $r=0$, on the other side of the computational grid, things are a little bit more complicated. As expected, the source of all our problems is related to the presence of $r$ in the denominator of some terms of (3.21), which blow up at $r=0$. Although the spin-2 fields $\Phi_{\lambda}$ are expected to be regular at $r=0$, the numerical implementation of the (singular at the origin) equations governing their evolution is a highly nontrivial task. In the following section we discuss the way we chose to numerically implement the system (3.21) at the origin.

It is worth mentioning that the precision of Python's floats limits the spatial resolution that can be used in our simulations. Specifically, it imposes an upper limit on the number of grid points we can use. This upper limit will be determined by the initial data as follows. The spatial resolution for which Python's double precision that we are using has been exceeded will mark the maximum number of grid points that can be used. This claim follows naturally from the fact that evolutions of initial data of higher resolution than that maximally allowed cannot be trusted as the numerical precision has already been exceeded on the initial slice. In the following the highest resolution that we are allowed to use is 1600 grid points in the nonhorizontal case (Sec. IV D 1) and 600 grid points in the horizontal case (Sec. IV D 2).

Now, in order to check the convergence of our numerical solutions, we define the convergence rate as follows,

$$
\mathrm{CR}=\frac{\log _{2}\left(E_{0} / E_{1}\right)}{\log _{2}\left(h_{0} / h_{1}\right)},
$$

where $E_{0}$ and $E_{1}$ are the normalized $l^{2}$ error norms for simulations of resolution $h_{0}$ and $h_{1}$, respectively. (Notice 
that $h_{0}<h_{1}$.) The errors $E$ will be computed against the numerical simulation of the highest resolution, which as discussed above will consist of 1600 grid points in the nonhorizontal case and 600 grid points in the horizontal case.

The code has been written in Python and is based on the Otago relativity group's conformal field equations solver, which has been appropriately amended and supplemented to fit the specific conformal problem we study in the present work.

\section{B. Treatment of the origin}

At first, the $1 / r$ and $1 / r^{2}$ terms must be expressed explicitly in (3.21). Observing (3.2) and (3.4) it is apparent that the quantities $g$ and $\rho$ introduce the singular terms in (3.21). As they stand, these terms are of the form $\csc \left(\frac{\pi r}{2}\right)$ and $\csc ^{2}\left(\frac{\pi r}{2}\right)$. To express them in a more manageable form, we introduce the maps

$$
\rho \mapsto S(r)-\frac{\kappa}{\sqrt{2} \pi r} \quad \text { and } \quad \frac{1}{g} \mapsto r C(r)+\frac{\kappa}{\pi},
$$

where $\kappa$ is the rescaling function (2.8) and $S, C$ are the regular functions

$$
S=\frac{r \kappa^{\prime}+\kappa-\pi r \kappa \cot (\pi r)}{\sqrt{2} \pi r} \quad \text { and } \quad C=\kappa\left(\csc (\pi r)-\frac{1}{\pi r}\right)
$$

with $S(0)=C(0)=0$. Expressing (3.21) through (4.2) in terms of $S$ and $C$, the $1 / r$ and $1 / r^{2}$ terms appear explicitly in (3.21).

In order to render the singular terms regular, certain conditions must be satisfied at $r=0$. These regularity conditions follow naturally from the system (3.21). There are two sets of regularity conditions obtained from the requirement that the coefficients of the terms $1 / r$ and $1 / r^{2}$ must vanish linearly and quadratically, respectively. Specifically, these conditions read

$$
\begin{aligned}
& \partial_{r} \Phi_{\lambda}=\left.0\right|_{r=0} \quad \text { and } \\
& \quad c_{1}(\lambda) \Phi_{\lambda}+c_{2}(\lambda) \Phi_{\lambda+1}+\left.c_{3}(\lambda) \Phi_{\lambda-1}\right|_{r=0}=0,
\end{aligned}
$$

where $\quad\left\{c_{1}, c_{2}, c_{3}\right\} \equiv\left\{\left(\lambda^{2}-4 \lambda-2\right)-\left(\alpha_{1,2}^{2}+\alpha_{2,3}^{2}\right) / 2\right.$, $\left.(4-\lambda) \alpha_{1,2}, \lambda \alpha_{2,3}\right\}$ with $\alpha_{x, y} \equiv \alpha_{(\lambda-x)(\lambda-y)}$ and $\lambda=0,1,2$, 3,4 . We must mention here that the $1 / r$ terms multiplied by the functions $C, S, \kappa^{\prime}$ are regular at the origin as these functions also vanish at $r=0$; thus, such terms do not contribute to the first regularity condition. Notice also that with (4.3) at hand, one can use l'Hopital's rule to evaluate the singular terms.

As it was mentioned in Sec. IV A, fourth-order central difference operators will be used to approximate the first and second derivatives at (and near) $r=0$. To do so, we have to introduce a couple of ghost points by extending the numerical grid to negative $r$. Now, in order to evaluate the components of the spin-2 field at the ghost points, we take advantage of the fact that the system (3.21) is symmetric under a simultaneous reflection $r \mapsto-r$ and spinor prime operation $\Phi_{\lambda}^{\prime}(t,-r) \mapsto \Phi_{4-\lambda}(t, r)$. Therefore, at the grid points $r_{0}, r_{1}, r_{2}$, the first spatial derivatives will be approximated by

$$
\begin{aligned}
& \partial_{r} \Phi_{\lambda}\left[r_{0}\right]=\frac{1}{12 h}\left(\Phi_{4-\lambda}\left[r_{2}\right]-8 \Phi_{4-\lambda}\left[r_{1}\right]+8 \Phi_{\lambda}\left[r_{1}\right]-\Phi_{\lambda}\left[r_{2}\right]\right), \\
& \partial_{r} \Phi_{\lambda}\left[r_{1}\right]=\frac{1}{12 h}\left(\Phi_{4-\lambda}\left[r_{1}\right]-8 \Phi_{4-\lambda}\left[r_{0}\right]+8 \Phi_{\lambda}\left[r_{2}\right]-\Phi_{\lambda}\left[r_{3}\right]\right), \\
& \partial_{r} \Phi_{\lambda}\left[r_{2}\right]=\frac{1}{12 h}\left(\Phi_{4-\lambda}\left[r_{0}\right]-8 \Phi_{\lambda}\left[r_{1}\right]+8 \Phi_{\lambda}\left[r_{3}\right]-\Phi_{\lambda}\left[r_{4}\right]\right),
\end{aligned}
$$

where $h$ is the grid spacing. Similarly, at the grid points $r_{1}$, $r_{2}$ (the point $r_{0}$ will be discussed separately in the following paragraph), the second spatial derivatives will be approximated by

$$
\begin{aligned}
\partial_{r}^{2} \Phi_{\lambda}\left[r_{1}\right]= & \frac{1}{12 h^{2}}\left(-\Phi_{4-\lambda}\left[r_{1}\right]+16 \Phi_{4-\lambda}\left[r_{0}\right]-30 \Phi_{\lambda}\left[r_{1}\right]\right. \\
& \left.+16 \Phi_{\lambda}\left[r_{2}\right]-\Phi_{\lambda}\left[r_{3}\right]\right), \\
\partial_{r}^{2} \Phi_{\lambda}\left[r_{2}\right]= & \frac{1}{12 h^{2}}\left(-\Phi_{4-\lambda}\left[r_{0}\right]+16 \Phi_{\lambda}\left[r_{1}\right]-30 \Phi_{\lambda}\left[r_{2}\right]\right. \\
& \left.+16 \Phi_{\lambda}\left[r_{3}\right]-\Phi_{\lambda}\left[r_{4}\right]\right) .
\end{aligned}
$$

Let us now describe the numerical implementation of the system (3.21) at $r=0$. Up to this point our numerical considerations have been quite standard. It turns out that in order to obtain stable and convergent numerical solutions, the second spatial derivatives and the terms $1 / r$ and $1 / r^{2}$ have to be treated in a special way at the origin.

Specifically, $\mathcal{F} / r$ terms with $\mathcal{F} \equiv\left\{C, S, \kappa^{\prime}\right\}$, where $C(0)=S(0)=\kappa^{\prime}(0)=0$, will be replaced in accordance with l'Hopital's rule, namely, $\mathcal{F} /\left.\left.r\right|_{r=0} \mapsto \partial_{r} \mathcal{F}\right|_{r=0}$. The remaining $1 / r$ terms are proportional to $\kappa^{2} \partial_{r} \Phi_{\lambda}$ and lead to the first regularity condition in (4.3). According to l'Hopital's rule, these terms can be evaluated in the following way: $\partial_{r} \Phi_{\lambda} /\left.\left.r\right|_{r=0} \mapsto \partial_{r}^{2} \Phi_{\lambda}\right|_{r=0}$. Approximating $\left.\partial_{r}^{2} \Phi_{\lambda}\right|_{r=0}$ with central difference operators as above [see (4.5)] did not lead to stable solutions. Interestingly, introducing the auxiliary functions $X_{\lambda} \equiv \partial_{r} \Phi_{\lambda}$, one can get stable and convergent numerical solutions by rewriting the second spatial derivatives in the form $\left.\partial_{r}^{2} \Phi_{\lambda}\right|_{r=0}=$ $\left.\partial_{r} X_{\lambda}\right|_{r=0}$ and approximating them by

$$
\begin{aligned}
\partial_{r}^{2} \Phi_{\lambda}\left[r_{0}\right]= & \frac{1}{12 h}\left(-X_{4-\lambda}\left[r_{2}\right]+8 X_{4-\lambda}\left[r_{1}\right]\right. \\
& \left.+8 X_{\lambda}\left[r_{1}\right]-X_{\lambda}\left[r_{2}\right]\right)
\end{aligned}
$$

where $X_{\lambda}^{\prime}(t,-r) \mapsto-X_{4-\lambda}(t, r)$ and $X_{\lambda}\left[r_{i}\right]=\partial_{r} \Phi_{\lambda}\left[r_{i}\right]$ are given by (4.4). Furthermore, it turns out that the stability of 
the solutions is guaranteed iff all the second spatial derivatives in (3.21), even the ones not arising from l'Hopital's rule, are approximated at $r=0$ by the above finite difference operator (4.6).

To evaluate the $1 / r^{2}$ terms at the origin, another set of auxiliary functions $\Upsilon_{\lambda} \equiv \Phi_{\lambda} / r$ must first be introduced. Subsequently, each $1 / r^{2}$ term can be written as
$\Phi_{\lambda} / r^{2}=\Upsilon_{\lambda} / r$, and the formerly quadratically singular terms now read $\left(c_{1} \Upsilon_{\lambda}+c_{2} \Upsilon_{\lambda+1}+c_{3} \Upsilon_{\lambda-1}\right) / r$. As before, by requiring the coefficient of $1 / r$ to vanish linearly, the second regularity condition in (4.3) can be expressed in the alternative form $c_{1} \Upsilon_{\lambda}+c_{2} \Upsilon_{\lambda+1}+\left.c_{3} \Upsilon_{\lambda-1}\right|_{r=0}=0$. Therefore, $1 / r^{2}$ will be evaluated at the origin according to the rule

$$
\left.\frac{c_{1} \Phi_{\lambda}+c_{2} \Phi_{\lambda+1}+c_{3} \Phi_{\lambda-1}}{r^{2}}\right|_{r=0} \mapsto c_{1} \partial_{r} \Upsilon_{\lambda}+c_{2} \partial_{r} \Upsilon_{\lambda+1}+\left.c_{3} \partial_{r} \Upsilon_{\lambda-1}\right|_{r=0}
$$

with the first spatial derivative being approximated by

$\partial_{r} \Upsilon_{\lambda}\left[r_{0}\right]=\frac{1}{12 h}\left(-\Upsilon_{4-\lambda}\left[r_{2}\right]+8 \Upsilon_{4-\lambda}\left[r_{1}\right]+8 \Upsilon_{\lambda}\left[r_{1}\right]-\Upsilon_{\lambda}\left[r_{2}\right]\right)$,

where $\Upsilon_{\lambda}^{\prime}(t,-r) \mapsto-\Upsilon_{4-\lambda}(t, r)$ and $\Upsilon_{\lambda}\left[r_{i}\right]=\Phi_{\lambda}\left[r_{i}\right] / r_{i}$.

\section{Initial data}

In accordance with the results of Sec. III C, the initial data must satisfy the conformal constraints (3.17) of the first-order PDE system and subsequently be evolved with (3.21). To do so, we first have to bring the constraints (3.17) into a more manageable form. Following [31], we construct initial data in terms of the $\Phi_{2}$ component of the spin-2 field, which can be freely specified, in a way such that no differential equations have to be solved.

The constraints (3.17) on the initial hypersurface, where $t=0$, reduce to

$$
2 \kappa \partial_{r} \Phi_{\lambda+1}-\alpha_{1,2} \pi C \Phi_{\lambda}-\frac{\alpha_{1,2} \kappa}{r} \Phi_{\lambda}-6 \sqrt{2} \pi S \Phi_{\lambda+1}+\frac{6 \kappa}{r} \Phi_{\lambda+1}-\alpha_{0,1} \pi C \Phi_{\lambda+2}-\frac{\alpha_{0,1} \kappa}{r} \Phi_{\lambda+2}=0,
$$

where $\alpha_{x, y} \equiv \alpha_{(\lambda-x)(\lambda-y)}$ and $\lambda=0,1,2$. Clearly, the system (4.7) is underdetermined and symmetric under the operation $\Phi_{\lambda}(0, r) \mapsto \Phi_{4-\lambda}(0, r)$. One way of solving (4.7) is by specifying freely two of the components of the spin-2 field and expressing the remaining three in terms of them. Alternatively, inspired by the aforementioned symmetry of (4.7), one can require that $\Phi_{\lambda}(0, r)=\Phi_{4-\lambda}(0, r)$ holds on the initial hypersurface. In this way, the unknowns have been reduced to three, and the independent equations constraining them have been reduced to two. Therefore, by specifying freely the, e.g., $\Phi_{2}$ component of the spin-2 field, a specific family of solutions of (4.7) can be obtained algebraically:

$$
\begin{aligned}
\Phi_{1}(0, r)= & \Phi_{3}(0, r)=\frac{\kappa x \partial_{r} \Phi_{2}+3 \pi(\kappa-\sqrt{2} S x) \Phi_{2}}{\alpha_{0} \pi(\kappa+x C)}, \\
\Phi_{0}(0, r)= & \Phi_{4}(0, r)=\frac{1}{\pi^{2} \alpha_{0} \alpha_{2}(\kappa+x C)^{3}}\left\{2 \kappa^{2} x^{2}(\kappa+x C) \partial_{r}^{2} \Phi_{2}\right. \\
& +2 \kappa x\left(x^{2}\left(C\left(\kappa^{\prime}-6 \sqrt{2} \pi S\right)-\kappa C^{\prime}\right)+6 \pi \kappa x(C-\sqrt{2} S)+7 \pi \kappa^{2}\right) \partial_{r} \Phi_{2} \\
& -6 \pi\left[\kappa x^{2}\left(C^{\prime}(\kappa-\sqrt{2} S x)+\sqrt{2} S^{\prime}(\kappa+x C)-(C+\sqrt{2} S) \kappa^{\prime}\right)\right. \\
& \left.\left.-\pi\left(6 C S^{2} x^{3}+6 \kappa S x^{2}(S-\sqrt{2} C)+\kappa^{2} x(2 C-7 \sqrt{2} S)+3 \kappa^{3}\right)+\frac{\pi}{6} \alpha_{0}^{2}(\kappa+x C)^{3}\right] \Phi_{2}\right\},
\end{aligned}
$$

where $x \equiv \pi r$. Hence, having specified the field $\Phi_{2}$ explicitly, the rest of the components of the spin-2 field can be computed algebraically from the system (4.8).

In the following, we will choose the field $\Phi_{2}$ to initially be a bump function of the form

$$
\Phi_{2}(0, r)= \begin{cases}\left(4 \frac{(r-a)(r-b)}{(b-a)^{2}}\right)^{16} & a \leq r \leq b \\ 0 & r>b \text { and } r<a\end{cases}
$$

centered at $r=(a+b) / 2$. Then the rest of the components of the spin-2 field are also bump functions and can be computed from (4.8). In this work we choose $a=0$ and $b=1$, which guarantees that the domain where (4.9) is nontrivial coincides with our computational domain, i.e., $0 \leq r \leq 1$, and that the initial data vanish at the boundary.

In addition, because the system (3.21) is second order in time, the values of the first temporal derivatives $\Psi_{\lambda}$ of the spin-2 field's components must also be specified on the 
initial hypersurface. The evolution equations (3.16) of the first-order system of PDEs, evaluated at $t=0$, will be used for this purpose. The values of the fields on the rhs of (3.16) can be evaluated from (4.8) and (4.9).

\section{Results}

The initial data constructed in the previous section will be evolved now with the system of second-order PDEs (3.21) in the two distinct conformal compactifications of Minkowski spacetime presented in Fig. 2. We report that our findings concerning the advantages of using the second-order system (3.21) instead of the first-order system of PDEs (3.16) to evolve the above initial data confirm the respective ones in $[28,30,31]$. Namely, evolutions with (3.21) lead to better accuracy and suppress the appearance of the high-frequency waves that travel against the characteristics and spoil the convergence of our numerical simulations.

\section{Nonhorizontal representation}

First, we present our results for the nonhorizontal case of Fig. 2(a), where $f=2 t$. The lowest nontrivial mode $l=2$ will be considered here. Thus, Eqs. (4.8) and (4.9) together with (3.16), evaluated at $t=0$, for the choice $l=2$ will be our initial data. We evolve these data with (3.21) using an explicit fourth-order Runge-Kutta scheme with a constant time step $\Delta t=\mathcal{C} h$, where $\mathcal{C}$ is the so-called CFL number. The results of the present section are obtained with $\mathcal{C}=0.05$. Recall also that the boundary at $r=1$ does not require boundary conditions as it is a total characteristic of (3.21), while the boundary at $r=0$ is treated in the way described in detail in Sec. IV B. The resulting numerical solutions for the components $\Phi_{0}$ and $\Phi_{4}$ of the spin-2 field are presented in Fig. 4. Clearly, $\Phi_{0}$ moves towards the origin, while $\Phi_{4}$ moves in the opposite direction towards the cylinder $I$ at $r=1$. The other components behave in a similar way.

Although, the numerical solutions displayed in Fig. 4 look quite smooth and stable, we have to conduct further tests to conclude with certainty that they are stable and convergent.

At first, we can look at their convergence rates (4.1). The behavior of the convergence rates with time for each component of the spin-2 field is illustrated in Fig. 5(a). It is clearly visible that during the evolution the convergence rates of all the components are a little bit above 4-a result that is in good agreement with the expected fourth-order convergence of our numerical scheme. This expectation follows naturally from the fact that the time integration is performed with a fourth-order Runge-Kutta method and the spatial derivatives are approximated with fourth-order finite difference operators.

Another way to test our numerical solutions is by checking to see if the vanishing of the constraint quantities (3.17) is satisfied during the evolution. As was mentioned previously, it is almost impossible not to violate the quantities (3.17) while numerically prescribing initial data on the initial hypersurface. We cannot avoid initially introducing some error into our system. Fortunately, as was proven at the end of Sec. III A 4, the structure of the subsidiary system of (3.17) does not allow these errors to grow exponentially during the evolution. Thus, we have to check if the numerical solutions of Fig. 4 confirm this expectation. To do so, we evaluate, at each time step, the three constraint quantities and compute their normalized $l^{2}$ norms along the whole computational domain. Figure 5(b) depicts the behavior of the three constraint quantities during the evolution. Taking into account that the initial violation of the constraints is, depending on the constraint quantity, between $10^{-9}$ and $10^{-8}$; then the data of Fig. 5(b) clearly indicate that the violation is contained at these levels

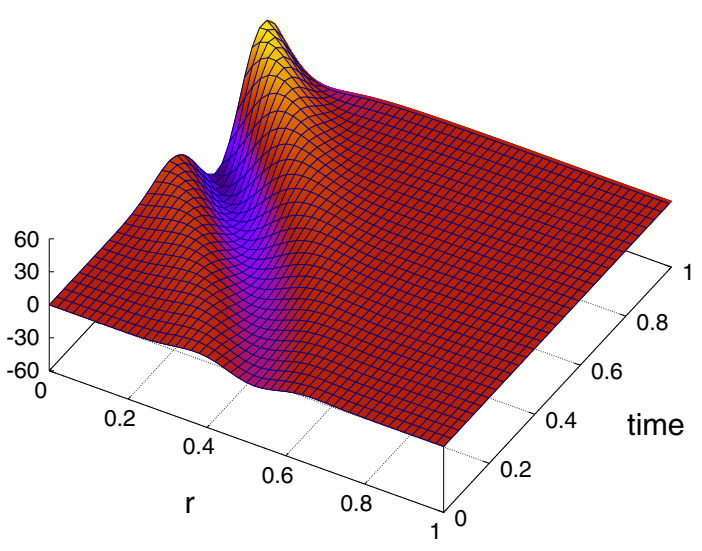

(a)

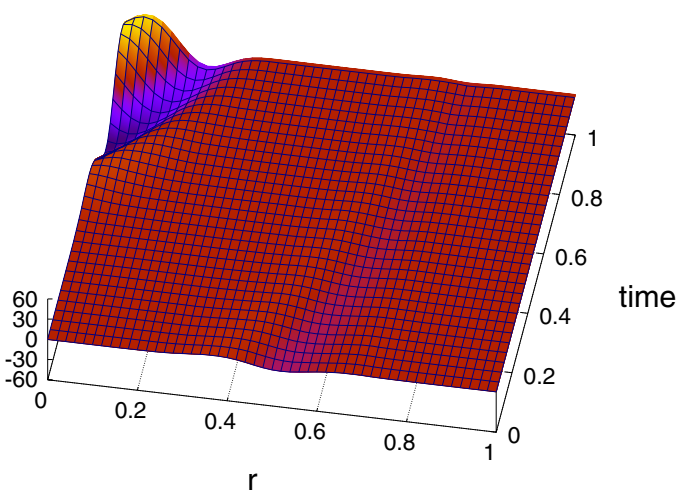

(b)

FIG. 4. The numerical solutions for (a) $\Phi_{0}$ and (b) $\Phi_{4}$ resulting from the evolution of the initial data (4.8) and (4.9) in the representation of Fig. 2(a). Notice that along the timeline of the origin, $\Phi_{0}(t, 0)=\Phi_{4}(t, 0)$ always holds. This is just a mere consequence of the symmetry of the system (3.21) under transformations of the form $\Phi_{\lambda}(t,-r) \mapsto \Phi_{4-\lambda}(t, r)$, which were used in Sec. IV B to regularize the singular terms of (3.21). 


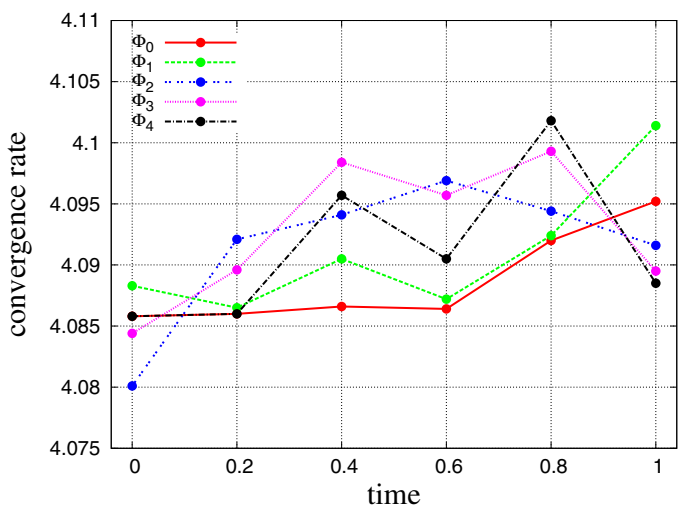

(a)



(b)

FIG. 5. For the numerical solutions of Fig. 4, we present the temporal evolution (a) of the convergence rates of each one of the components of the spin-2 field and (b) of the violation of the vanishing of the constraint quantities (3.17). The critical set $I^{+}$at $t=1 \mathrm{can}$ be successfully reached, but as expected, we cannot go beyond it; i.e., we always maintain $t<1$.

during the evolution. This extremely pleasant feature is a consequence of the linear nature of (3.21) and of the comparatively short period of evolution. In addition, the convergence of the constraint quantities with increasing resolution has also been checked. Our findings agree with the expected fourth-order convergence to zero.

It is expected that logarithmic singularities will develop when our numerical simulations reach the critical set $I^{+}$at $t=1$. [Recall that beyond $I^{+}$the domain of nonhyperbolicity of (3.21) starts; see Fig. 3(a).] Our findings confirm this expectation. Namely, because we use an explicit Runge-Kutta scheme, $I^{+}$can be successfully reached, but going beyond this leads immediately to instabilities and code crash. This means that the part of Minkowski spacetime beyond $t=1$ cannot be covered by our numerical simulations. So, it is not possible to cover the whole of Minkowski spacetime in the representation of Fig. 2(a). But, there is a possibility to achieve this in the horizontal representation of Fig. 2(b) discussed in the following section.

\section{Horizontal representation}

Let us now turn to the horizontal representation of Fig. 2(b), where the whole of Minkowski spacetime is represented as a conformally equivalent region of the Einstein static universe with a rectangular shape in the chosen coordinates. This representation is numerically quite advantageous as the critical set $I^{+}$, future null $\mathcal{I}^{+}$and timelike $i^{+}$infinity are located at the same time slice $t=1$. Therefore, with the last time step, which takes us to $t=1$, we not only reach $I^{+}$but also $\mathcal{I}^{+}$and $i^{+}$. Thus, we do not have to go beyond $t=1$ to cover the whole Minkowski spacetime. But this comes at a price: The speed of the characteristic curves at $t=1$ [see Fig. 3(b)] becomes infinite - which makes our endeavor to reach $t=1$ extremely challenging. In the following we investigate numerically the possibility of reaching $t=1$ in this setting.
As above, our initial data consist of (4.8) and (4.9) together with (3.16), evaluated at $t=0$, for the lowest nontrivial mode $l=2$ and the choice (2.16). Again, we use an explicit fourth-order Runge-Kutta scheme of constant time step with CFL number $\mathcal{C}=0.1$. The resulting numerical solutions for the components $\Phi_{0}$ and $\Phi_{4}$ of the spin-2 field are presented in Fig. 6. Notice that while $\Phi_{0}$ and $\Phi_{4}$ move slowly towards the origin and the cylinder, respectively, at late times this indolent movement is accelerated. A look at the characteristic curves of Fig. 3(b) suffices to explain this behavior. The rapid shift of the orientation of the characteristics from almost vertical to almost parallel at late times is responsible for the observed increase of the propagation speed of the evolved data. The remaining components behave in a similar way.

By inspection of Fig. 6, the obtained solutions are evidently smooth and stable during the whole evolution. At first sight, this seemingly doubtful result, which implies that our numerical solutions are smooth and stable even at $t=1$, where the characteristic speed becomes infinity, can be attributed to the explicit Runge-Kutta scheme we are using and to the extremely steep characteristics of the horizontal representation [see Fig. 3(b)]. Because of the former, the evolution equations are actually never evaluated at $t=1$. The latter now delays the violation of the CFL condition, resulting from the increase of the characteristic speed, to very late times. When appropriately combined, these two features can lead to the smooth and stable solutions of Fig. 6. It is noteworthy that when the characteristic curves are less steep than the ones we are using, $t=1$ cannot be reached in a stable way and the solutions blow up there. So, the steeper the characteristic curves are, the better our numerical results. The numerical factor in (2.16) controls the steepness of the characteristics; this explains why the specific value appearing in (2.16) was chosen.

To study the convergence properties of the solutions of Fig. 6, we take a look at their convergence rates (4.1). 


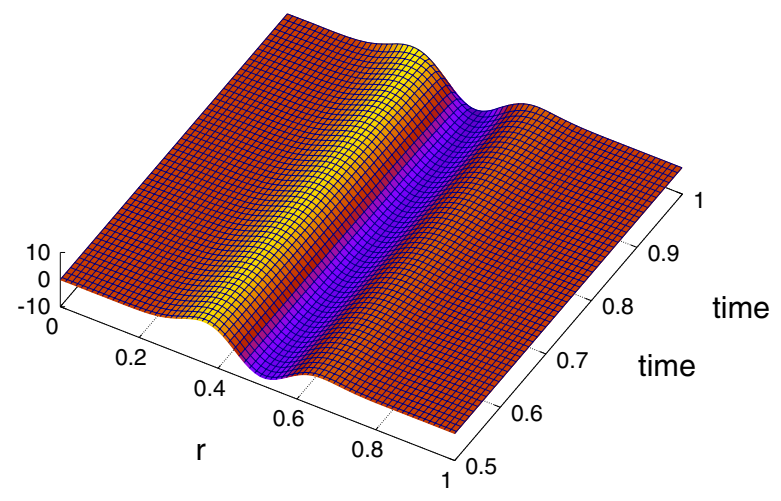

(a)



(b)

FIG. 6. The numerical solutions for (a) $\Phi_{0}$ and (b) $\Phi_{4}$ resulting from the evolution of the initial data (4.8) and (4.9) in the representation of Fig. 2(b). Notice the increase of the propagation speed at late times $t \approx 1$ attributed to the abrupt shift of the orientation of the characteristic curves there [see Fig. 3(b)].

Figure 7(a) illustrates the convergence rates for each component of the spin-2 field as a function of time. We have zoomed in to the interesting region where $t \rightarrow 1$, as for earlier times $t<0.992$ the convergence rates are well above 4. For late times we lose convergence while approaching $t=1$ and end up with convergence rates close to unity at $t=1$. The observed loss of convergence can be ascribed to the violation of the CFL condition caused by the increase of the characteristic speed at late times [see Fig. 3(b)]. Thus, by decreasing the $\mathrm{CFL}$ number $\mathcal{C}$, the loss of convergence can be significantly reduced and postponed to even later times, but it cannot be avoided altogether as we approach $t=1$ where the characteristic speed becomes infinite.

Now, let us study the behavior of the vanishing of the constraint quantities (3.17) during the evolution. Figure 7(b) depicts the temporal evolutions of the constraint quantities $C_{\lambda}$ with time. The initial violation of the constraints is of the order of $10^{-7}$ and is maintained at this level, as can be seen in Fig. 7(b), until the quite late time $t \approx 0.999$. Thereafter, the constraints are increasingly violated. This is again a consequence of the violation of the CFL condition that follows from the increase of the characteristic speed while approaching $t=1$. A decrease of the $\mathrm{CFL}$ number $\mathcal{C}$ delays the phase of increasing violation of the constraints but cannot suppress it.

Recall that all our results in the present section have been obtained using a time integrator that marches at a constant pace throughout the evolution. So, when using a constant time step, our findings above show that, although our numerical solutions do not blow up at $t=1, t=1$ cannot be reached without a loss of convergence and a considerable violation of the constraints that are caused by the unavoidable increase of the characteristic speed while $t \rightarrow 1$. If the latter is really the reason for the

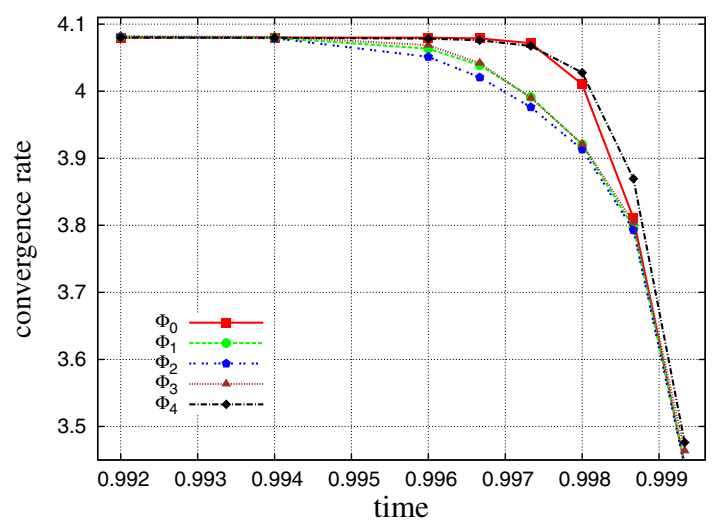

(a)

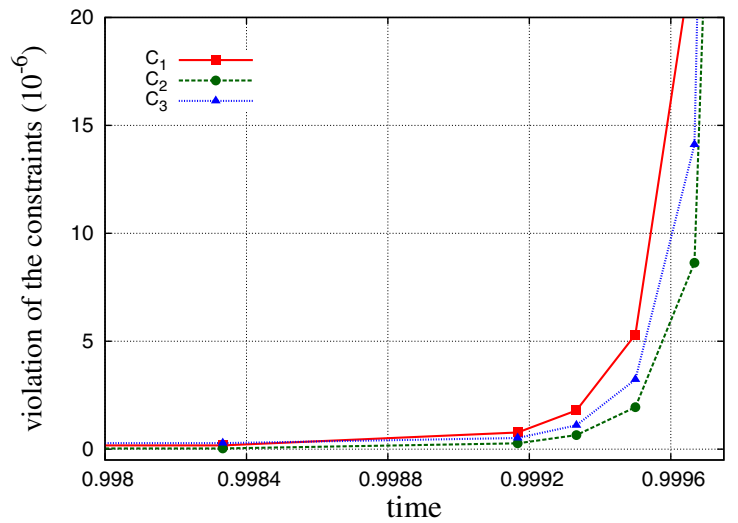

(b)

FIG. 7. For the numerical solutions of Fig. 6, the time dependence (a) of the convergence rates of each one of the components of the spin-2 field and (b) of the violation of the vanishing of the constraint quantities (3.17) is presented. The observed loss of convergence and nonpreservation of the constraints is caused by the rapid increase of the characteristic speed at late times, which in turn leads to the violation of the CFL condition. 


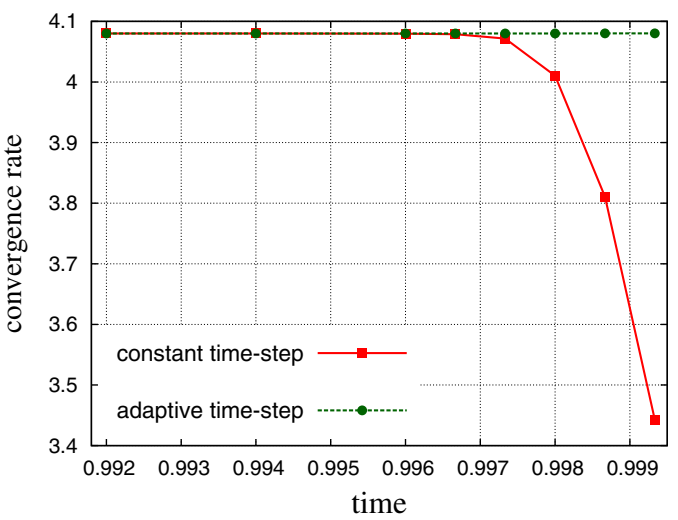

(a)



(b)

FIG. 8. For the numerical solutions of Fig. 6, we present the behavior with time (a) of the convergence rate of the worst converging component $\Phi_{0}$ of the spin-2 field and (b) of the violation of the vanishing of the mostly violated constraint quantity $C_{1}$. In each figure, we compare the results of Fig. 7 obtained with a constant time step to the ones obtained with an adaptive time step. Obviously, the use of an adaptive time step restores the respective quantities to their expected values and enables us to reach $t=1$ at a distance of merely $10^{-12}$.

underperformance of our code close to $t=1$, then by using an explicit Runge-Kutta scheme with an adaptive time step, $t=1$ could be approached arbitrarily close without losing convergence and increasingly violating the constraints. A time integrator of this type adjusts the time step and, consequently, the CFL number $\mathcal{C}$, according to the magnitude of the characteristic speed. in a way that the CFL condition is always satisfied. In Fig. 8 the convergence rates and the behavior of the constraint quantities (3.17) resulting from an evolution with an adaptive time step are compared to the corresponding results of Fig. 7 obtained with a constant time step. Clearly, the use of an adaptive time step restores the convergence rates and the preservation of the constraints to their expected values. In addition, for the simulation of highest resolution, here 600 grid points, we managed to advance to $t \approx 0.999999999999$, just $10^{-12}$ from $t=1$. We could not get closer to $t=1$ as the time step becomes of the order of $10^{-17}$, exceeding Python's double precision. In principle, using higher quadruple or octuple precision can get us even closer to $t=1$.

\section{DISCUSSION}

In this work it was shown that the generalized conformal field equations [25] can be used to study gravitational perturbations on the whole of Minkowski spacetime $\mathbb{M}$ and not only in the regions close to spacelike infinity $i^{0}$.

This has been achieved by slightly diverging from Friedrich's original formulation where spacelike infinity $i^{0}$ is first placed at the origin by a coordinate inversion and then blown up to a cylinder by an appropriate rescaling of the resulting spacetime. Here, a different strategy was followed. Instead of inverting the coordinates, we first conformally compactified $\mathbb{M}$ into the Einstein static universe $\mathbb{E}$ (see Sec. II A), and then by appropriately rescaling the resulting conformal metric (2.4), spacelike infinity was blown up to a cylinder in the spirit of Friedrich [see (2.7)]. The free functions $\kappa(r)$ and $f(t)$, introduced by the rescaling, control the shape and the location of $i^{0}$ and $\mathcal{I}$. Here, we chose to work in the two representations of Fig. 2. The former representation is an example of the general setting where the temporal positions of $i^{+}, i^{0}$ and $\mathcal{I}^{+}$on $\mathbb{M}$ are distinct, while the latter is quite special as all of $i^{+}, i^{0}, \mathcal{I}^{+}$are positioned on the same time slice.

The cost of including the whole of $\mathbb{M}$ into the computational domain is that some terms of the generalized conformal field equations, in both their representation as a system of first-order (3.16) and second-order (3.21) PDEs, are singular at $r=0$. Note, however, that this is not due to a deficiency of the conformal rescaling but to the use of polar coordinates adapted to the spherical symmetry of the background Minkowski spacetime. Therefore, although the spin-2 field is regular at the origin, the numerical implementation of the equations governing its dynamics is highly involved. In Sec. IV B the numerical implementation of the system (3.21) at the origin is described in detail.

In the nonhorizontal representation of Fig. 2(a), it is possible to reach the critical set $I^{+}$at $t=1$ without loss of convergence and with the constraint quantities (3.17) preserved (see Sec. IVD 1). This was made possible because of the finite speed of the characteristic curves at $I^{+}$[see Fig. 3(a)] and because of the explicit Runge-Kutta scheme we are using. But any attempt to go beyond $I^{+}$, i.e., to enter the domain of nonhyperbolicity of (3.21), leads to code crash almost immediately, as expected. Therefore, it is not possible to cover parts of $\mathbb{M}$ lying beyond the time slice $t=1$ in this representation.

In the horizontal representation of Fig. 2(b), the whole of Minkowski spacetime has been restricted conformally between the time slices $t=-1$ and $t=1$ (which are, in fact, null hypersurfaces) that go through the critical sets $I^{-}$ 
and $I^{+}$, respectively. This feature leaves open the possibility of performing a global simulation of $\mathbb{M}$, namely, evolving data from past $i^{-}$all the way to future timelike infinity $i^{+}$. This possibility was extensively investigated in Sec. IV D 2; the main source of our difficulties here is related to the fact that the speed of the characteristics becomes infinite at $t=1$ [see Fig. 3(b)]. A constant time step throughout the evolution can get us to $t=1$ in a smooth way (see Fig. 6) but with a considerable loss of convergence and violation of the constraints (see Fig. 7). We can get around this by using an adaptive time step. In this case how close we can get to $t=1$ depends on the available computational precision. With Python's double precision we managed to approach $t=1$ to a distance of only $10^{-12}$ with the expected fourth-order convergence and preservation of the constraints (see Fig. 8).

Having shown that our formulation can be successfully applied for the Minkowski spacetime, we can move on and apply it to spacetimes subject to less restrictive symmetry conditions.

\section{ACKNOWLEDGMENTS}

J. F. would like to thank the Department of Mathematics at the University of Oslo for hospitality. Part of this research was supported by the European Research Council through the FP7-IDEAS-ERC Starting Grant scheme, Project No. 278011 STUCCOFIELDS.
[1] B. P. Abbott et al. (LIGO Scientific Collaboration and Virgo Collaboration), Observation of Gravitational Waves from a Binary Black Hole Merger, Phys. Rev. Lett. 116, 061102 (2016).

[2] F. Pretorius, Evolution of Binary Black Hole Spacetimes, Phys. Rev. Lett. 95, 121101 (2005).

[3] M. Campanelli, C. O. Lousto, P. Marronetti, and Y. Zlochower, Accurate Evolutions of Orbiting Black-Hole Binaries without excision, Phys. Rev. Lett. 96, 111101 (2006).

[4] J. G. Baker, J. Centrella, D. Choi, M. Koppitz, and J. van Meter, Gravitational-Wave Extraction from an Inspiraling Configuration of Merging Black Holes, Phys. Rev. Lett. 96, 111102 (2006).

[5] A. Zenginoğlu, A hyperboloidal study of tail decay rates for scalar and Yang-Mills fields, Classical Quantum Gravity 25, 175013 (2008).

[6] H. Bondi, M. G. J. van der Burg, and A. W. K. Metzner, Gravitational waves in General Relativity. VII. Waves from axi-symmetric isolated systems, Proc. R. Soc. A, 269, 21 (1962).

[7] R. K. Sachs, Gravitational waves in General Relativity. VIII. Waves in asymptotically flat space-time, Proc. R. Soc. A, 270, 103 (1962).

[8] J. Winicour, Characteristic evolution and matching, Living Rev. Relativ. 15,2 (2012).

[9] R. Gómez, R. L. Marsa, and J. Winicour, Black hole excision with matching, Phys. Rev. D 56, 6310 (1997).

[10] C. J. Handmer, B. Szilágyi, and J. Winicour, Gauge invariant spectral Cauchy characteristic extraction, Classical Quantum Gravity 32, 235018 (2015).

[11] C. J. Handmer, B. Szilágyi, and J. Winicour, Spectral Cauchy characteristic extraction of strain, news and gravitational radiation flux, Classical Quantum Gravity 33, 225007 (2016).

[12] R. Penrose, Asymptotic Properties of Fields and SpaceTimes, Phys. Rev. Lett. 10, 66 (1963).
[13] H. Friedrich, On the regular and the asymptotic characteristic initial value problem for Einstein's vacuum field equations, Proc. R. Soc. A, 375, 169 (1981).

[14] P. Hübner, From now to timelike infinity on a finite grid, Classical Quantum Gravity 18, 1871 (2001).

[15] J. Frauendiener, Numerical treatment of the hyperboloidal initial value problem for the vacuum Einstein equations: III. On the determination of radiation, Classical Quantum Gravity 17, 373 (2000).

[16] J. Frauendiener and M. Hein, Numerical evolution of axisymmetric, isolated systems in General Relativity, Phys. Rev. D 66, 124004 (2002).

[17] V. Moncrief and O. Rinne, Regularity of the Einstein equations at future null infinity, Classical Quantum Gravity 26, 125010 (2009).

[18] O. Rinne and V. Moncrief, Hyperboloidal Einstein-matter evolution and tails for scalar and Yang-Mills fields, Classical Quantum Gravity 30, 095009 (2013).

[19] O. Rinne, Formation and decay of Einstein-Yang-Mills black holes, Phys. Rev. D 90, 124084 (2014).

[20] O. Rinne, An axisymmetric evolution code for the Einstein equations on hyperboloidal slices, Classical Quantum Gravity 27, 035014 (2010).

[21] A. V.Viñuales, Ph.D. thesis, University Illes Balears, 2015, arXiv:1512.00776.

[22] A. Zenginoğlu, Hyperbolodial evolution with the Einstein equations, Classical Quantum Gravity 25, 195025 (2008).

[23] G. Doulis and O. Rinne, Numerical construction of initial data for Einstein's equations with static extension to spacelike infinity, Classical Quantum Gravity 33, 075014 (2016).

[24] G. Doulis and O. Rinne, Numerical Brill-Lindquist initial data with a Schwarzschildean end at spatial infinity, J. Phys. Conf. Ser. 600, 012039 (2015).

[25] H. Friedrich, Gravitational fields near space-like and null infinity, J. Geom. Phys. 24, 83 (1998).

[26] A. Zenginoğlu, Ph.D. thesis, University of Potsdam, 2007, arXiv:0711.0873. 
[27] F. Beyer, G. Doulis, J. Frauendiener, and B. Whale, Numerical space-times near space-like and null infinity. The spin-2 system on Minkowski space, Classical Quantum Gravity 29, 245013 (2012).

[28] G. Doulis and J. Frauendiener, The second order spin-2 system in flat space near space-like and null-infinity, Gen. Relativ. Gravit. 45, 1365 (2013).

[29] F. Beyer, G. Doulis, J. Frauendiener, and B. Whale, Linearized gravitational waves near space-like and null infinity, Springer Proc. Math. Stat. 60, 3 (2014).

[30] F. Beyer, G. Doulis, J. Frauendiener, and B. Whale, The spin-2 equation on Minkowski background, Springer Proc. Math. Stat. 60, 465 (2014).

[31] G. Doulis, Ph.D. thesis, University of Otago, 2013 (http:// hdl.handle.net/10523/4088).
[32] C. Stevens, Ph.D. thesis, University of Otago (2016).

[33] H. O. Kreiss and O.E. Ortiz, Finite element and finite difference methods for hyperbolic partial differential equations, Lect. Notes Phys. 604, 359 (2002).

[34] S. W. Hawking and G. F. R. Ellis, The Large Scale Structure of Space-Time, 1st ed. (Cambridge University Press, Cambridge, England, 1973).

[35] H. Friedrich, Spin-2 fields on Minkowski space near spacelike and null infinity, Classical Quantum Gravity 20, 101 (2003).

[36] R. Penrose, Zero rest-mass fields including gravitation: Asymptotic behaviour, Proc. R. Soc. A 284, 159 (1965).

[37] R. Penrose and W. Rindler, Spinors and Space-Time, 1st ed. (Cambridge University Press, Cambridge, England, 1986), Vols. 1 and 2. 